\title{
AN OPERATOR-THEORETIC APPROACH TO TRUNCATED MOMENT PROBLEMS
}

\author{
RAÚL E. CURTO \\ Department of Mathematics, The University of Iowa \\ Iowa City, Iowa 52242, U.S.A. \\ E-mail: curto@math.uiowa.edu
}

\begin{abstract}
We survey recent developments in operator theory and moment problems, beginning with the study of quadratic hyponormality for unilateral weighted shifts, its connections with truncated Hamburger, Stieltjes, Hausdorff and Toeplitz moment problems, and the subsequent proof that polynomially hyponormal operators need not be subnormal. We present a general elementary approach to truncated moment problems in one or several real or complex variables, based on matrix positivity and extension. Together with the construction of a "functional calculus" for the columns of the associated moment matrix, our operator-theoretic approach allows us to obtain existence theorems for the truncated complex moment problem, in case the columns satisfy one of several natural constraints. We also include an application to the Riemann-quadrature problem from numerical analysis.
\end{abstract}

1. Quadratic hyponormality and the Subnormal Completion Problem. Let $\mathcal{H}$ be a complex Hilbert space and let $\mathcal{L}(\mathcal{H})$ be the algebra of bounded operators on $\mathcal{H}$. An operator $T \in \mathcal{L}(\mathcal{H})$ is said to be normal if $T^{*} T=T T^{*}$, hyponormal if $T^{*} T \geq T T^{*}$, and subnormal if $T=\left.N\right|_{\mathcal{H}}$, where $N$ is normal on some Hilbert space $\mathcal{K} \supseteq \mathcal{H}$. If $T$ is subnormal, then $T$ is also hyponormal: For, if $N=\left(\begin{array}{ll}T & A \\ 0 & B\end{array}\right)$ is a normal extension of $T$, we have

$$
0=N^{*} N-N N^{*}=\left(\begin{array}{cc}
T^{*} & 0 \\
A^{*} & B^{*}
\end{array}\right)\left(\begin{array}{cc}
T & A \\
0 & B
\end{array}\right)-\left(\begin{array}{cc}
T & A \\
0 & B
\end{array}\right)\left(\begin{array}{cc}
T^{*} & 0 \\
A^{*} & B^{*}
\end{array}\right)
$$

1991 Mathematics Subject Classification: Primary 47A20, 44A60, 47B20; Secondary 47-02, 15A57, 47B37, 65D32.

This survey is based on four lectures given at the Stefan Banach International Mathematical Center during April 5-8, 1994, as part of the Semester on Linear Operators. The author is deeply indebted to the organizers, Professors J. Janas, F. H. Szafraniec and J. Zemánek, for their invitation and for the warm hospitality during his stay in Warsaw.

Research partially supported by a grant from the National Science Foundation.

The paper is in final form and no version of it will be published elsewhere. 


$$
=\left(\begin{array}{cc}
T^{*} T-T T^{*}-A A^{*} & * \\
* & *
\end{array}\right),
$$

so that $T^{*} T-T T^{*}=A A^{*} \geq 0$. The converse is false, although examples are not entirely easy to construct, and require $\operatorname{dim} \mathcal{H}=\infty$.

The notions of hyponormality and subnormality were introduced by P. R. Halmos in the early 50's [Hal]. On one hand, hyponormality reflects the geometric nature of the notion of normality, with the corresponding implications in terms of matrix positivity; on the other hand, subnormality is intimately related to the notion of analyticity for complex functions, through the restriction of the functional calculus to invariant subspaces. For the construction of models, hyponormality needs singular integrals and multiplication operators on Sobolev spaces, subnormality requires Cauchy transforms and complex function theory. Subnormality does imply hyponormality, but the substantial distance between the two notions is precisely what has caused the two theories to follow separate courses.

Subnormality is invariant under polynomial calculus (since for a polynomial $p, p(S)=$ $\left.p(N)\right|_{\mathcal{H}}$, and $p(N)$ is still normal), but the square of a hyponormal operator may not be hyponormal. It is then natural to consider the class of polynomially hyponormal operators (those operators which remain hyponormal under polynomial calculus), which obviously contains all subnormal operators. Whether these two classes coincide remained unknown for over thirty-five years, and served as motivation for many of the ideas to be discussed here.

Problem 1.1. Must a polynomially hyponormal operator be necessarily subnormal?

For a historical account of this problem, the reader is referred to [Cu3]. Here we will emphasize that both polynomial hyponormality and subnormality are related to hyponormality through two discrete bridges, discussed in detail in [Cu2]. Since Problem 1 is intrinsically an infinite-dimensional problem, one is naturally led to consider the class of unilateral weighted shifts as a source of examples. Moreover, S. McCullough and V. Paulsen $[\mathrm{McCP}]$ proved that in order to solve Problem 1.1 it suffices to consider the class of weighted shifts.

Recall that given a sequence of positive numbers $\alpha: \alpha_{0}, \alpha_{1}, \ldots$ (called weights), the unilateral weighted shift $W_{\alpha}$ associated with $\alpha$ is the operator on $\ell^{2}\left(\mathbb{Z}_{+}\right)$defined by $W_{\alpha} e_{n}:=\alpha_{n} e_{n+1}$ (all $n \geq 0$ ), where $\left\{e_{n}\right\}_{n=0}^{\infty}$ is the canonical orthonormal basis for $\ell^{2}$; the moments of $\alpha$ are given by $\gamma_{0}:=1, \gamma_{n+1}:=\alpha_{n}^{2} \gamma_{n}(n \geq 0)$. It is straightforward to check that $W_{\alpha}$ can never be normal, and that $W_{\alpha}$ is hyponormal if and only if $\alpha_{n} \leq \alpha_{n+1}$ for all $n \geq 0$. We wish to exhibit a fundamental 3-way relationship among operator theory, matrix theory and measure theory. First, recall the Bram-Halmos criterion for subnormality, which says that an operator $T$ is subnormal if and only if

$$
\sum_{i, j}\left(T^{i} x_{j}, T^{j} x_{i}\right) \geq 0
$$

for all finite collections $x_{0}, x_{1}, \ldots, x_{k} \in \mathcal{H}$ ([Bra], [Con, III.1.9]). Using the Choleski algorithm for operator matrices, it is easy to see that this is equivalent to the positivity of the matrices $\left(T^{* j} T^{i}-T^{i} T^{* j}\right)_{i, j=1}^{k}$, for $k=1,2, \ldots$ If we denote by $[A, B]:=A B-B A$ the 
commutator of two operators $A$ and $B$, and if we define $T$ to be $k$-hyponormal whenever the $k \times k$ operator matrix $M_{k}(T):=\left(\left[T^{* j}, T^{i}\right]\right)_{i, j=1}^{k}$ is positive, then the Bram-Halmos criterion can be rephrased as saying that $T$ is subnormal if and only if $T$ is $k$-hyponormal for every $k \geq 1$ ([CMX]).

Secondly, C. Berger's characterization of subnormality for unilateral weighted shifts states that $W_{\alpha}$ is subnormal if and only if the sequence $\left\{\gamma_{n}\right\}$ can be interpolated by a probability measure $\mu$ supported on the interval $\left[0, \sup _{n}\left|\alpha_{n}\right|\right]$, i.e.,

$$
\gamma_{n}=\int t^{n} d \mu(t) \quad(\text { for all } n \geq 0)
$$

[Con III.8.16]); briefly said, $W_{\alpha}$ is subnormal if and only if the moments of $\alpha$ are the moments of some probability measure $\mu$. This immediately establishes a connection between unilateral weighted shifts and the classical theory of moments, which has been quite useful.

While subnormality is related to a moment problem, $k$-hyponormality for weighted shifts admits a matricial characterization, as follows: $W_{\alpha}$ is $k$-hyponormal if and only if $\left(\gamma_{n+i+j}\right)_{i, j=0}^{k}$ is positive for all $n \geq 0$ ([Cu1], [Cu2]). When combined with the BramHalmos criterion, one finds that $W_{\alpha}$ is subnormal if and only if the matrices $\left(\gamma_{n+i+j}\right)_{i, j=0}^{k}$ are positive for all $n \geq 0$ and for all $k \geq 1$, which can be seen to be equivalent to the positivity of the two infinite matrices $\left(\gamma_{i+j}\right)_{i, j=0}^{\infty}$ and $\left(\gamma_{i+j+1}\right)_{i, j=0}^{\infty}$. By the classical result of Stieltjes, this is in turn equivalent to the existence of a probability measure supported on $[0,+\infty)$ which interpolates the sequence $\left\{\gamma_{n}\right\}$. This provides a new proof of Berger's Theorem, and completes the third bridge among operator theory, matrix theory and measure theory. Thus, a subnormal shift corresponds to two positive Hankel matrices, which in turn correspond to a compactly supported measure on $[0,+\infty)$.

The matricial criterion for $k$-hyponormality provides, in addition, a technique for distinguishing between $k$-hyponormality and $(k+1)$-hyponormality, and it is particularly helpful in the study of recursively generated weighted shifts ([CuF1], [CuF2]). In an effort to distinguish between subnormality and polynomially hyponormality, one considers first the classes of 2-hyponormal and quadratic hyponormal weighted shifts. As we have already mentioned,

$$
W_{\alpha} \text { is 2-hyponormal } \Leftrightarrow\left(\begin{array}{ccc}
\gamma_{n} & \gamma_{n+1} & \gamma_{n+2} \\
\gamma_{n+1} & \gamma_{n+2} & \gamma_{n+3} \\
\gamma_{n+2} & \gamma_{n+3} & \gamma_{n+4}
\end{array}\right) \geq 0 \quad(\text { for all } n \geq 0) .
$$

To study quadratic hyponormality, however, we must resort to a completely different scheme. If $W_{\alpha}$ is hyponormal, each upper-left-hand corner of the infinite matrix $\left[\left(W_{\alpha}+\right.\right.$ $\left.\left.s W_{\alpha}^{2}\right)^{*}, W_{\alpha}+s W_{\alpha}^{2}\right]$ must be nonnegative. Now, observe that such a corner is of the form

$$
P_{n}\left[\left(W_{\alpha}+s W_{\alpha}^{2}\right)^{*}, W_{\alpha}+s W_{\alpha}^{2}\right] P_{n}=\left(\begin{array}{cccccc}
q_{0} & \bar{r}_{0} & 0 & \cdots & 0 & 0 \\
r_{0} & q_{1} & \bar{r}_{1} & \cdots & 0 & 0 \\
0 & r_{0} & q_{2} & \cdots & 0 & 0 \\
\ldots & \ldots & \ldots & \ldots & \ldots \\
0 & 0 & 0 & \cdots & q_{n-1} & \bar{r}_{n-1} \\
0 & 0 & 0 & \cdots & r_{n-1} & q_{n}
\end{array}\right),
$$


where $P_{n}$ is the orthogonal projection onto the subspace generated by $\left\{e_{0}, \ldots, e_{n}\right\}$,

$$
\begin{gathered}
q_{n}:=u_{n}+|s|^{2} v_{n}, \quad r_{n}:=s \sqrt{w_{n}}, \quad u_{n}:=\alpha_{n}^{2}-\alpha_{n-1}^{2}, \\
v_{n}:=\alpha_{n}^{2} \alpha_{n+1}^{2}-\alpha_{n-1}^{2} \alpha_{n-2}^{2}, \quad w_{n}:=\alpha_{n}^{2}\left(\alpha_{n+1}^{2}-\alpha_{n-1}^{2}\right)^{2},
\end{gathered}
$$

and, for notational convenience, $\alpha_{-2} \equiv \alpha_{-1}:=0$. The determinant $d_{n}$ of this tri-diagonal matrix satisfies the following 2 -step recursive formula:

$$
d_{0}=q_{0}, \quad d_{1}=q_{0} q_{1}-\left|r_{0}\right|^{2}, \quad d_{n+2}=q_{n+2} d_{n+1}-\left|r_{n+1}\right|^{2} d_{n} ;
$$

if we let $t:=|s|^{2}$, we observe that $d_{n}$ is a polynomial in $t$ of degree $n+1$, and if we write $d_{n}=\sum_{i=0}^{n+1} c(n, i) t^{i}$, then the coefficients $c(n, i)$ satisfy a double-indexed recursive formula, namely

$$
\begin{gathered}
c(n+2, i)=u_{n+2} c(n+1, i)+v_{n+2} c(n+1, i-1)-w_{n+1} c(n, i-1), \\
c(n, 0)=u_{0} \ldots u_{n}, \quad c(n, n+1)=v_{0} \ldots v_{n}, \quad c(1,1)=u_{1} v_{0}+v_{1} u_{0}-w_{0}
\end{gathered}
$$

$(n \geq 0, i \geq 1)$. Using (1.1) and (1.2) in a judicious manner, one can obtain a number of results about quadratic hyponormality.

For 2-hyponormality, we can use the above mentioned matricial characterization to see at once that when $W_{\alpha}$ is hyponormal and $\alpha_{n}=\alpha_{n+1}$ for some $n$, then $W_{\alpha}$ is 2 -hyponormal if and only if $W_{\alpha}$ is flat, that is, $\alpha_{1}=\alpha_{2}=\ldots$ (In [Sta], J. Stampfli has previously established this for subnormal shifts, so our result showed that the assumed "rigidity" really pertains to 2-hyponormality.) Concerning weak 2-hyponormality, A. Joshi proved in [Jos1] and [Jos2] that the shift with weights $\alpha_{0}=\alpha_{1}=a, \alpha_{2}=\alpha_{3}=\ldots=b$, $0<a<b$, is not quadratically hyponormal, and later P. Fan [Fan] established that for $a=1, b=2$, and $0<s<\sqrt{5} / 5, W_{\alpha}+s W_{\alpha}^{2}$ is not hyponormal. With the aid of symbolic manipulation, and the recursive relations for $d_{n}$, it was shown in ([Cu1], [Cu2]) that a hyponormal weighted shift with three equal weights can't be quadratically hyponormal without being flat. A natural question then arises: Can a quadratically hyponormal shift have two equal weights without being flat?

The existence of such shifts was established in [Cu2], and it led to an essential distinction between 2-hyponormality and quadratic hyponormality, which eventually became the starting point for an inductive procedure to separate subnormality from polynomial hyponormality.

Connected with the above example is the problem of finding adequate descriptions of quadratic hyponormality. For instance, one would like to parameterize all quadratically hyponormal shifts whose first two weights are equal to 1. Symbolic manipulation shows that there are no such shifts with $1<\alpha_{2}=\alpha_{3}$ [Cu1, Proposition 11], that $\alpha_{2}$ is always less than $\sqrt{2}$, and that $\alpha_{3} \geq\left(2-\alpha_{2}^{2}\right)^{-1 / 2}$.

Problem 1.2. Describe all quadratically hyponormal weighted shifts with $\alpha_{0}=$ $\alpha_{1}=1$.

There is another class of unilateral weighted shifts that has played a key role in the recent solution of Problem 1.1. We are referring to the class of recursively generated weighted shifts, especially those known as "abc" type, which we now proceed to describe. Back in 1966, Stampfli [Sta] showed that for arbitrary $\alpha_{0}<\alpha_{1}<\alpha_{2}$ there always exists 
a subnormal unilateral weighted shift $T$ whose first three weights are $\alpha_{0}, \alpha_{1}$, $\alpha_{2}$; he also proved that given four or more weights, it may not be possible to find a subnormal completion. Stampfli's proofs were of "geometric" nature, in the sense that he built the normal extension directly out of the weights $\alpha_{0}, \alpha_{1}$ and $\alpha_{2}$. An alternative way to establish this is to assume that a 2-atomic interpolating measure exists, and then to use symbolic manipulation to analyze and solve the system of 4 equations in 4 unknowns

$$
\left\{\begin{array}{l}
\rho_{0}+\rho_{1}=\gamma_{0} \\
\rho_{0} s_{0}+\rho_{1} s_{1}=\gamma_{1} \\
\rho_{0} s_{0}^{2}+\rho_{1} s_{1}^{2}=\gamma_{2} \\
\rho_{0} s_{0}^{3}+\rho_{1} s_{1}^{3}=\gamma_{3}
\end{array}\right.
$$

which has a unique solution when $\alpha_{0}<\alpha_{1}<\alpha_{2}$. The subnormal completion corresponds then to the measure $\mu:=\rho_{0} \delta_{s_{0}}+\rho_{1} \delta_{s_{1}}$. In an effort to understand why this is true, and why four weights may not necessarily admit a subnormal completion, one is naturally led to the following problem.

Problem 1.3 (Subnormal Completion Problem). Given an initial segment of weights $\alpha: \alpha_{0}, \ldots, \alpha_{m}$, find necessary and sufficient conditions for the existence of $\widehat{\alpha} \in \ell^{\infty}\left(\mathbb{Z}_{+}\right)$ such that $\widehat{\alpha}_{i}=\alpha_{i}(i=0, \ldots, m)$ and $W_{\hat{\alpha}}$ is subnormal. Equivalently, find necessary and sufficient conditions for the existence of a compactly supported probability measure $\mu$ on $[0,+\infty)$ which interpolates $\gamma_{0}, \ldots, \gamma_{m+1}$, i.e.,

$$
\int t^{n} d \mu(t)=\gamma_{n} \quad(0 \leq n \leq m+1)
$$

The answer is surprisingly simple, and it involves the positivity of two Hankel matrices. The Subnormal Completion Criterion, obtained in [CuF1], distinguishes two cases, according to whether $m$ is even or odd. In the former case, say $m=2 k$, there exists a subnormal completion if and only if

$$
A(k):=\left(\begin{array}{cccc}
\gamma_{0} & \gamma_{1} & \ldots & \gamma_{k} \\
\gamma_{1} & \gamma_{2} & \ldots & \gamma_{k+1} \\
\ldots \ldots & \ldots \ldots \ldots & \ldots \\
\gamma_{k} & \gamma_{k+1} & \ldots & \gamma_{2 k}
\end{array}\right) \geq 0, \quad B(k):=\left(\begin{array}{cccc}
\gamma_{1} & \gamma_{2} & \ldots & \gamma_{k+1} \\
\gamma_{2} & \gamma_{3} & \ldots & \gamma_{k+2} \\
\ldots \ldots & \ldots \ldots \ldots & \ldots & \ldots \\
\gamma_{k+1} & \gamma_{k+2} & \ldots & \gamma_{2 k+1}
\end{array}\right) \geq 0
$$

and the vector $\mathbf{v}(k+1, k):=\left(\gamma_{k+1} \gamma_{k+2} \ldots \gamma_{2 k+1}\right)^{T}$ belongs to the range of the matrix $A(k)$. When $m=2 k-1$, the criterion requires that the matrix $A(k)$ be positive, that

$$
B(k-1):=\left(\begin{array}{cccc}
\gamma_{1} & \gamma_{2} & \ldots & \gamma_{k} \\
\gamma_{2} & \gamma_{3} & \ldots & \gamma_{k+1} \\
\ldots \ldots \ldots \ldots \ldots & \ldots \ldots \\
\gamma_{k} & \gamma_{k+1} & \ldots & \gamma_{2 k-1}
\end{array}\right) \geq 0
$$

and that the vector $\mathbf{v}(k+1, k-1):=\left(\gamma_{k+1} \gamma_{k+2} \ldots \gamma_{2 k}\right)^{T}$ belong to the range of $B(k-1)$.

One consequence of our approach is that if $\alpha: \alpha_{0}, \ldots, \alpha_{m}$ admits a subnormal completion, then it admits one whose associated measure $\mu$ is finitely atomic, i.e., supp $\mu$ is a finite set. This completion is recursive, i.e., there exist scalars $\varphi_{0}, \ldots, \varphi_{r-1}$ such that

$$
\gamma_{n+r}=\varphi_{0} \gamma_{n}+\ldots+\varphi_{r-1} \gamma_{n+r-1}
$$

for all $n \geq 0$. The coefficients $\varphi_{0}, \ldots, \varphi_{r-1}$ give rise to the generating function

$$
g(t):=t^{r}-\left(\varphi_{0}+\ldots+\varphi_{r-1} t^{r-1}\right) \quad(t \in \mathbb{R}) .
$$


Lemma 1.4 ([CuF1, Theorem 3.9], [CuF2, Proposition 3.3]). g has $r$ distinct real roots $0 \leq s_{0}<\ldots<s_{r-1}$.

Let

$$
V:=\left(\begin{array}{cccc}
1 & 1 & \ldots & 1 \\
s_{0} & s_{1} & \ldots & s_{r-1} \\
\ldots \ldots & \ldots \ldots \ldots & \ldots \\
s_{0}^{r-1} & s_{1}^{r-1} & \ldots & s_{r-1}^{r-1}
\end{array}\right)
$$

$V$ is invertible, and if we let

$$
\left(\begin{array}{c}
\rho_{0} \\
\cdots \\
\rho_{r-1}
\end{array}\right):=V^{-1}\left(\begin{array}{c}
\gamma_{0} \\
\cdots \\
\gamma_{r-1}
\end{array}\right)
$$

then $\mu:=\rho_{0} \delta_{s_{0}}+\ldots+\rho_{0} \delta_{s_{r-1}}$ is positive and interpolates $\alpha$.

Stampfli's case is obtained when $m=2$ : here $\varphi_{0}=-\alpha_{0}^{2} \alpha_{1}^{2}\left(\alpha_{2}^{2}-\alpha_{1}^{2}\right) /\left(\alpha_{1}^{2}-\alpha_{0}^{2}\right)$ and $\varphi_{1}=\alpha_{1}^{2}\left(\alpha_{2}^{2}-\alpha_{0}^{2}\right) /\left(\alpha_{1}^{2}-\alpha_{0}^{2}\right)$, from which one can easily obtain $s_{0}$ and $s_{1}$, and a fortiori $\rho_{0}$ and $\rho_{1}$ (cf. [Cu3, p. 40], [CuF2]).

It is possible to solve the Subnormal Completion Problem by first looking for a measure $\mu$ on $\mathbb{R}$ such that $\int t^{k} d \mu(t)=\gamma_{k}(0 \leq k \leq m+1)$ (this is the so-called truncated Hamburger moment problem) and then establishing that the support must lie in $[0,+\infty)$. As a way of motivating what we will later do in the case of truncated complex moment problems, we introduce here a "functional calculus" viewpoint to study truncated Hamburger moment problems.

Given $\gamma: \gamma_{0}, \ldots, \gamma_{m+1}$, we let $k:=[(m+1) / 2]$, and we form the square matrix $A(k):=\left(\gamma_{j+\ell}\right)_{j, \ell=0}^{k}$. First observe that if $\mu$ satisfies $(1.3)$ and $p(t) \equiv \sum_{j=0}^{k} a_{j} t^{j}$, then

$$
0 \leq \int|p(t)|^{2} d \mu(t)=\sum_{j, \ell=0}^{k} a_{j} \bar{a}_{\ell} \int t^{j+\ell} d \mu(t)=\sum_{j, \ell=0}^{k} a_{j} \bar{a}_{\ell} \gamma_{j+\ell}=(A(k) \widehat{p}, \widehat{p}),
$$

where $\widehat{p}:=\left(a_{0}, \ldots, a_{k}\right) \in \mathbb{C}^{k+1}$. This induces us to regard the expression $(A(k) \widehat{p}, \widehat{p})$ as $\langle p, p\rangle_{A(k)}$; the positivity of $A(k)$ (which is easily read from (1.4)) implies that $\langle\cdot, \cdot\rangle_{A(k)}$ is a positive semidefinite inner product, giving rise to a genuine inner product in the associated quotient structure $\mathbb{C}[t] / \sim$. In order to even consider the operator of multiplication by $t$ in $\mathbb{C}[t] / \sim$, one must first be able to extend $A(k)$ to a bigger matrix, with similar properties. This requires a structure theorem, which we know present.

THEOREM 1.5 (Structure theorem for positive Hankel matrices [CuF3, Theorem 2.4]. Given a positive Hankel matrix $A(k) \equiv\left(\gamma_{j+\ell}\right)_{j, \ell=0}^{k}$, let $r:=\operatorname{rank} A(k)$. Then

(i) $A(r-1):=\left(\gamma_{j+\ell}\right)_{j, \ell}^{r-1}$ is positive and invertible;

(ii) every entry of $A(k)$, with the possible exception of $\gamma_{2 k}$, is given recursively in terms of the entries in $A(r-1)$; concretely, if $r \leq k$ and $\left(\varphi_{0} \ldots \varphi_{r-1}\right)^{T}:=$ $A(r-1)\left(\gamma_{r} \ldots \gamma_{2 r-1}\right)^{T}$, then

$$
\gamma_{r+s}=\varphi_{0} \gamma_{s}+\ldots+\varphi_{r-1} \gamma_{r+s-1} \quad(0 \leq s \leq 2 k-r-1) .
$$

If we label the columns of $A(k)$ as $1, T, \ldots, T^{k}$, condition (ii) in Theorem 1.5 says 
that

$$
T^{r}=\varphi_{0} 1+\ldots+\varphi_{r-1} T^{r-1}
$$

and that we can formally multiply $(1.5)$ by $T^{s}$ to produce

$$
T^{r+s}=\varphi_{0} T^{s}+\ldots+\varphi_{r-1} T^{r+s-1} \quad(0 \leq s \leq k-r-1) .
$$

This phenomenon is what we call recursiveness, and allows us to focus attention on the associated generating function $g(t):=t^{r}-\left(\varphi_{0}+\ldots+\varphi_{r-1} t^{r-1}\right)$. Any measure $\mu$ solving (1.3) will necessarily have support in the zero set of $g$. An easy dimensioncounting argument shows that $\operatorname{supp} \mu=\mathcal{Z}(g)$, so once the roots of $g$ are found (which automatically become the atoms $s_{0}<\ldots<s_{r-1}$ of $\left.\mu\right)$, we can obtain the densities $\rho_{0}, \ldots, \rho_{r-1}$ by solving the Vandermonde system $V\left(\begin{array}{llll}\rho_{0} & \ldots & \rho_{r-1}\end{array}\right)^{T}=\left(\begin{array}{lll}\gamma_{0} & \ldots & \gamma_{r-1}\end{array}\right)^{T}$. To go from a solution of the truncated Hamburger moment problem to a solution of the truncated Stieltjes moment problem, that is, $\operatorname{supp} \mu \subseteq[0,+\infty)$, one resorts to the associated matrices $B(k)$ or $B(k-1)$ (depending upon the parity of $m$ ). What essentially happens is that the condition $B(k) \geq 0$ (or $B(k-1) \geq 0)$ translates into $\langle t p, p\rangle_{A(k)} \geq 0$, which then implies that $s_{0} \geq 0$.

Our techniques are elementary and general, and they also allow us to obtain solutions to the truncated Hausdorff and Toeplitz moment problems (cf. [CuF3]). In this section, however, we are mainly interested in the applications to unilateral weighted shifts, which will lead us to the conclusion that the classes of quadratically hyponormal and 2-hyponormal shifts are indeed quite different. We shall return to moment problems in Section 3.

As mentioned before, there is a simple characterization of 2-hyponormality ( $W_{\alpha}$ is 2-hyponormal if and only if $\left(\gamma_{n+i+j}\right)_{i, j=0}^{2} \geq 0$ for every $\left.n \geq 0\right)$, but the same cannot be said of quadratic hyponormality. As a matter of fact, here are two of the main problems still unresolved in this topic.

Problem 1.6. Is there a characterization of quadratic hyponormality along the lines of the above mentioned characterization of 2-hyponormality?

Problem 1.7. Find models for quadratic hyponormality.

One approach to the second problem is to think of quadratically hyponormal shifts as perturbations of subnormal shifts, and to recall that these are norm-limits of recursively generated shifts [CuF1, Theorem 4.2]; thus, one is led to the consideration of perturbations of recursive subnormal shifts, those given by finitely atomic measures on $[0,+\infty)$. A concrete situation is the following

Problem 1.8. Assume that $\alpha_{0}<\alpha_{1}<\alpha_{2}$ are given. For which $x$ 's is $W_{x\left(\alpha_{0}, \alpha_{1}, \alpha_{2}\right)}$ quadratically hyponormal?

(By $W_{x\left(\alpha_{0}, \alpha_{1}, \alpha_{2}\right)^{\text {c }}}$ we mean the shift whose weights are calculated according to the recursive relation $\alpha_{n+1}=\varphi_{1}+\varphi_{0} \frac{1}{\alpha_{n}^{2}}$, where $\varphi_{0}=-\frac{a b(c-b)}{b-a}$ and $\varphi_{1}=\frac{b(c-a)}{b-a} ; W_{\left(\alpha_{0}, \alpha_{1}, \alpha_{2}\right)}$, which coincides with the shift constructed by Stampfli in [Sta], is subnormal, and we perturb it by inserting $x$ as the zero-th weight.) 
To start, we would like to find the range of $x$ 's for which $W_{x\left(\alpha_{0}, \alpha_{1}, \alpha_{2}\right)^{-}}$is 2-hyponormal. By a special case of the Extension Principle ([CuF2, Theorems 3.7 and 3.10]), this happens precisely when the shift $W_{x\left(\alpha_{0}, \alpha_{1}, \alpha_{2}\right)^{)}}$is subnormal, or equivalently when $x^{2} \leq\left(\rho_{0} / s_{0}+\right.$ $\left.\rho_{1} / s_{1}\right)^{-1}\left[\mathrm{CuF} 2\right.$, Theorem 3.10], where $\mu=\rho_{0} \delta_{s_{0}}+\rho_{1} \delta_{s_{1}}$ is the measure associated to $W_{x\left(\alpha_{0}, \alpha_{1}, \alpha_{2}\right)}$. For the example $a=1, b=2, c=3$, one finds that the corresponding shift is 2-hyponormal if and only if $x^{2} \leq \frac{2}{3}$.

For quadratic hyponormality, the actual calculation of the range of $x$ 's is much more difficult, and it involves heavy use of symbolic manipulation.

Theorem 1.9 (Quadratic Hyponormality Criterion ([CuF2, Theorem 4.3]). Let

$$
\begin{aligned}
& H_{2}:=\sup \left\{x>0: W_{x\left(\alpha_{0}, \alpha_{1}, \alpha_{2}\right)^{-}} \text {is 2-hyponormal }\right\} \\
& h_{2}^{+}:=\sup \left\{x>0: W_{x\left(\alpha_{0}, \alpha_{1}, \alpha_{2}\right)^{-}} \text {has } c(n, i) \geq 0 \text { for all } n, i\right\},
\end{aligned}
$$

and write $a:=\alpha_{0}^{2}, b:=\alpha_{1}^{2}, c:=\alpha_{2}^{2}$. Then

1) $H_{2}=\left(\frac{\varphi_{0}}{a-\varphi_{1}}\right)^{1 / 2}=\left(\frac{a b(c-b)}{(b-a)^{2}+b(c-b)}\right)^{1 / 2}$;

2) $h_{2}^{+}=\left(\frac{a^{2} b^{2} c+a b^{2}(c-a) K+a b(c-b) K^{2}}{a^{3} b+a b(c-a) K+\left(a^{2}+b c-2 a b\right) K^{2}}\right)^{1 / 2}$,

where $K:=-\varphi_{1}^{2} L^{2} / \varphi_{0}$, with $L:=\left\|W_{\left(\alpha_{0}, \alpha_{1}, \alpha_{2}\right)^{2}}\right\|=\left(\left(\varphi_{1}+\sqrt{\varphi_{1}^{2}+4 \varphi_{0}}\right) / 2\right)^{1 / 2}$, and

3) $H_{2}<h_{2}^{+}$.

When $a=1, b=2$ and $c=3$, we get $h_{2}^{+} \cong 0.8563$ and $H_{2}=\sqrt{\frac{2}{3}} \cong 0.8165$. Of course the most important of the three statements is the last one, since it tells us that no matter how we choose a, $b$ and $c$, we can always find $x$ 's (a whole interval of them!) such that the shift $W_{x\left(\alpha_{0}, \alpha_{1}, \alpha_{2}\right)^{-}}$is quadratically hyponormal and not 2-hyponormal. Similar techniques can also be used to show that there exists $\epsilon>0$ with the following property: For every $1<\alpha_{1}<1+\epsilon$ there exists $\alpha_{2}>\alpha_{1}$ such that $W_{1\left(1, \alpha_{1}, \alpha_{2}\right)^{\circ}}$ is quadratically hyponormal. (Recall that $W_{1\left(1, \alpha_{1}, \alpha_{2}\right)^{-}}$can't be 2-hyponormal.) This shows that non-trivial quadratically hyponormal shifts with two equal weights are quite common, and deserve to be fully classified (Problem 1.2).

2. Existence of non-subnormal polynomially hyponormal operators. In addition to providing many concrete examples of non-subnormal quadratically hyponormal operators, the above criterion gives strong evidence that the classes of polynomially hyponormal and subnormal operators are different. Actually, and since 2-hyponormality and subnormality have identical moduli for $W_{x\left(\alpha_{0}, \alpha_{1}, \alpha_{2}\right)^{-}}[\mathrm{CuF} 2$, Section 3], the results seem to indicate that perhaps something much stronger is true, namely that the classes of polynomially hyponormal and 2-hyponormal operators are different. The following theorem provides an answer to the stronger problem, and it therefore solves Problem 1.1.

Theorem 2.1 ([CuP1], [CuP2, Theorem 2.1]). There exists a polynomially hyponormal operator $T$ which is not 2-hyponormal.

By combining this with the main result in $[\mathrm{McCP}]$ one gets at once the following result. 
Corollary 2.2 ([CuP2, Corollary 2.8]). There exists a unilateral weighted shift which is polynomially hyponormal and not subnormal.

We briefly indicate below the main technical steps leading to the solution of Problem 1.1; details can be found in [CuP2]. First, we recall that Agler [Agl] found in 1985 a 1-1 correspondence between contractions with a cyclic vector and certain linear functionals on $\mathbb{C}[z, \bar{z}]$. If $T \in \mathcal{L}(\mathcal{H}),\|T\| \leq 1, \gamma \in \mathcal{H}$, and $p \in \mathbb{C}[z, \bar{z}], p(z, \bar{z})=\sum_{m, n} a_{m n} z^{m} \bar{z}^{n}$, we define the so-called hereditary functional calculus by letting $p\left(T, T^{*}\right):=\sum_{m, n} a_{m n} T^{* n} T^{m}$, and a linear functional $\Lambda_{T}: \mathbb{C}[z, \bar{z}] \rightarrow \mathbb{C}$ by $\Lambda_{T}(p):=\left(p\left(T, T^{*}\right) \gamma, \gamma\right), p \in \mathbb{C}[z, \bar{z}] . \Lambda_{T}$ has two important properties: (i) $\Lambda_{T}(p \bar{p}) \geq 0$, and (ii) $\Lambda_{T}((1-z \bar{z}) p \bar{p}) \geq 0$. Conversely, if $\Lambda: \mathbb{C}[z, \bar{z}] \rightarrow \mathbb{C}$ is a linear functional satisfying (i) and (ii), we let $\mathcal{N}:=\{p \in \mathbb{C}[z]$ : $\Lambda(p \bar{p})=0\}$, and observe that $\Lambda \mathcal{N} \subseteq \mathcal{N}$, and that $\mathbb{C}[z] / \mathcal{N}$ is a pre-Hilbert space with the inner product $\langle p, q\rangle:=\Lambda(p \bar{q}), p, q \in \mathbb{C}[z]$. Moreover, $z \mathcal{N} \subseteq \mathcal{N}$. If we now let $T:=M_{z}$ on $\mathcal{H}:=(\mathbb{C}[z] / \mathcal{N})$, we see that $T$ is a contraction operator with cyclic vector $1+\mathcal{N}$.

Next, we recall $([\mathrm{CuP} 2])$ that for $T$ cyclic with vector $\gamma$,

$$
\begin{aligned}
T \text { is 2-hyponormal } & \Leftrightarrow\left(\begin{array}{ccc}
I & T^{*} & T^{* 2} \\
T & T^{*} T & T^{* 2} T \\
T^{2} & T^{*} T^{2} & T^{* 2} T^{2}
\end{array}\right) \geq 0 \\
& \Leftrightarrow\left(\left(\begin{array}{ccc}
I & T^{*} & T^{* 2} \\
T & T^{*} T & T^{* 2} T \\
T^{2} & T^{*} T^{2} & T^{* 2} T^{2}
\end{array}\right)\left(\begin{array}{c}
p_{0}(T) \gamma \\
p_{1}(T) \gamma \\
p_{2}(T) \gamma
\end{array}\right),\left(\begin{array}{c}
p_{0}(T) \gamma \\
p_{1}(T) \gamma \\
p_{2}(T) \gamma
\end{array}\right)\right) \geq 0 \\
& \Leftrightarrow\left(\left|p_{0}+p_{1} \bar{z}+p_{2} \bar{z}^{2}\right|^{2}\left(T, T^{*}\right) \gamma, \gamma\right) \geq 0 \\
& \Leftrightarrow \Lambda_{T}\left(\left|p_{0}+p_{1} \bar{z}+p_{2} \bar{z}^{2}\right|^{2}\right) \geq 0 \quad\left(p_{0}, p_{1}, p_{2} \in \mathbb{C}[z]\right) .
\end{aligned}
$$

Similarly,

$$
\begin{aligned}
T \text { is polynomially hyponormal } & \Leftrightarrow\left(\begin{array}{cc}
I & r(T)^{*} \\
r(T) & r(T)^{*} r(T)
\end{array}\right) \geq 0 \quad(r \in \mathbb{C}[z]) \\
& \left.\Leftrightarrow\left(\begin{array}{cc}
I & r(T)^{*} \\
r(T) & r(T)^{*} r(T)
\end{array}\right),\left(\begin{array}{c}
p(T) \gamma \\
q(T) \gamma
\end{array}\right),\left(\begin{array}{c}
p(T) \gamma \\
q(T) \gamma
\end{array}\right)\right) \geq 0 \\
& \Leftrightarrow\left(|p+q \bar{r}|^{2}\left(T, T^{*}\right) \gamma, \gamma\right) \geq 0 \\
& \Leftrightarrow \Lambda_{T}\left(|p+q \bar{r}|^{2}\right) \geq 0 \quad(p, q, r \in \mathbb{C}[z]) .
\end{aligned}
$$

We are thus led to consider two cones of polynomials: $\mathcal{S}^{2}$, generated by all polynomials of the form $(1-z \bar{z})|p|^{2}$ and $\left|p_{0}+p_{1} \bar{z}+p_{2} \bar{z}^{2}\right|^{2}$, and $\mathcal{W}$, generated by those of the form $(1-z \bar{z})|p|^{2}$ and $|p+q \bar{r}|^{2}$. The above calculations show that $T$ is 2 -hyponormal if and only if $\left.\Lambda_{T}\right|_{\mathcal{S}^{2}} \geq 0$, and that $T$ is polynomially hyponormal if and only if $\left.\Lambda_{T}\right|_{\mathcal{W}} \geq 0$. From this viewpoint, Problem 1.1 will be resolved if we can accomplish the following

GoAL. Find $\Lambda: \mathbb{C}[z, \bar{z}] \rightarrow \mathbb{C}$ and $p \in \mathcal{S}^{2}$ such that $\Lambda_{T} \mid \mathcal{W} \geq 0$ and $\Lambda(p)<0$.

Once this linear functional has been found, we can build $T$ in such a way that $\Lambda_{T}=\Lambda$. To construct $\Lambda$, we introduce some auxiliary cones. First, we shall denote by $\Gamma$ the cone generated by polynomials of the form $|p+q \bar{r}|^{2}$, and for $m \geq 0$, we shall denote by $\mathbb{C}[z, \bar{z}]_{m}$ the cone of polynomials whose total degree in $z$ and $\bar{z}$ is at most $m$, by $\mathbb{C}[z, \bar{z}]^{h}$ the collection of homogeneous polynomials, and by $\mathbb{C}[z, \bar{z}]_{m}^{h}$ the set of homogeneous poly- 
nomials of degree $m$ (with similar definitions for $\mathbb{R}[x, y]_{m}, \mathbb{R}[x, y]^{h}$ and $\mathbb{R}[x, y]_{m}^{h}$ ). Finally, for $K$ a cone in $\mathbb{C}[z, \bar{z}]$, we shall let $K_{m}:=K \cap \mathbb{C}[z, \bar{z}]_{m}, K^{h}:=K \cap \mathbb{C}[z, \bar{z}]^{h}$, and $K_{m}^{h}:=K \cap \mathbb{C}[z, \bar{z}]_{m}^{h}$. Observe that $\Gamma$ is smaller than $\mathcal{W}$, but easier to handle; our strategy will exploit this, together with the fact that $\Gamma_{4}^{h}$ is actually equal to $\mathcal{W}_{4}^{h}$.

The first observation is that $\left\{p(z, \bar{z}) \in \mathbb{C}[z, \bar{z}]_{m}^{h}: p=\bar{p}\right\}=\mathbb{R}[x, y]_{m}^{h}$, via the usual identification $(z+\bar{z}) / 2=x,(z-\bar{z}) /(2 i)=y$, so that it suffices to construct a real linear functional $\Lambda$ on $\mathbb{R}[x, y]$. Next, we recall that if $E$ is a (real, finite dimensional) vector space, if $K$ is a convex subset of $E$ with $\operatorname{int}(K) \neq \emptyset$, and if $M$ is a linear manifold in $E$ such that $M \cap \operatorname{int}(K)=\emptyset$, then there exists a hyperplane $H \supseteq M$ such that $H \cap \operatorname{int}(K)=\emptyset$ (cf. [CoC, I.3.1.3]).

To build $\Lambda$, we plan to use the fact that quadratic hyponormality and 2-hyponormality are far apart, and therefore it should be possible to separate $\mathcal{S}^{2}$ from $\mathcal{W}_{4}$. For technical reasons, it is more convenient to consider $\Gamma_{4}^{h}$ first. Thus, we shall attempt to define $\Lambda_{4}^{h}: \mathbb{R}[x, y]_{4}^{h} \rightarrow \mathbb{R}$ such that $\left.\Lambda_{4}^{h}\right|_{\Gamma_{4}^{h}} \geq 0,\left.\Lambda_{4}^{h}\right|_{\operatorname{int}\left(\Gamma_{4}^{h}\right)}>0$, and $\Lambda_{4}^{h}(p)<0$ for some $p \in$ $\mathcal{S}^{2} \cap \mathbb{R}[x, y]_{4}^{h}$. Once this is done, we shall try and extend $\Lambda_{4}^{h}$ to $\mathbb{R}[x, y]_{4}$, then to $\mathbb{R}[x, y]_{5}$, to $\mathbb{R}[x, y]_{6}$, etc. The corresponding convex sets to be considered are $\Gamma_{4}^{h}, \mathcal{W}_{4}^{h}, \mathcal{W}_{4}, \mathcal{W}, \mathcal{W}_{6}$, etc. Two results are needed to make the extension strategy work: on one hand, $\operatorname{int}\left(\Gamma_{4}^{h}\right)$, $\operatorname{int}\left(\mathcal{W}_{4}^{h}\right), \operatorname{int}\left(\mathcal{W}_{4}\right), \operatorname{int}\left(\mathcal{W}_{5}\right), \operatorname{int}\left(\mathcal{W}_{6}\right)$, etc., must all be nonempty; on the other hand, we must verify that $\mathcal{W}_{4}^{h}=\Gamma_{4}^{h}$. This is accomplished in Steps 1 and 3 below; Step 2 is required in the actual construction of $\Lambda_{4}^{h}$.

STEP 1 ([CuP2, Lemma 2.3]).

(i) For $m \geq 0, \mathbb{R}[x, y]_{m}=\mathcal{W}_{m}-\mathcal{W}_{m}\left(\Rightarrow \operatorname{int}\left(\mathcal{W}_{m}\right) \neq \emptyset\right)$.

(ii) For $m \geq 0$ even, $\mathbb{R}[x, y]_{m}=\Gamma_{m}-\Gamma_{m}=\mathcal{W}_{m}-\mathcal{W}_{m}$.

(iii) For $m \geq 0$ even, $\mathbb{R}[x, y]_{m}^{h}=\Gamma_{m}^{h}-\Gamma_{m}^{h}\left(\Rightarrow \operatorname{int}\left(\Gamma_{m}^{h}\right) \neq \emptyset\right)$.

SteP 2. ([CuP2, Lemma 2.4]). $\Gamma_{4}$ is generated by all polynomials of the form

$$
\left|c_{0}+c_{1} z+c_{2} \bar{z}+c_{3} z^{2}+c_{4} z \bar{z}+c_{5} \bar{z}^{2}\right|^{2},
$$

where $c_{4}=0$ or $c_{5}=0$.

STEP $3\left(\left[\mathrm{CuP} 2,\left[\right.\right.\right.$ Lemma 2.6]). $\mathcal{W}_{4}^{h} \subseteq \Gamma_{4}^{h}$, that is, the $\left(1-|z|^{2}\right) \sum_{j}\left|s_{j}(z)\right|^{2}$ component of a homogeneous polynomial of total degree 4 can be eliminated.

Step $4\left(\left[\mathrm{CuP} 2\right.\right.$, Lemma 2.5]). Define $\Lambda_{4}^{h}: \mathbb{R}[x, y]_{4}^{h} \rightarrow \mathbb{R}$ by

$$
\Lambda_{4}^{h}\left(z^{4}\right):=0, \quad \Lambda_{4}^{h}\left(z^{3} \bar{z}\right):=b, \quad \Lambda_{4}^{h}\left(z^{2} \bar{z}^{2}\right):=1, \quad \Lambda_{4}^{h}\left(z \bar{z}^{3}\right):=b, \quad \Lambda_{4}^{h}\left(\bar{z}^{4}\right):=0 .
$$

Then

$$
\begin{aligned}
\Lambda_{4}^{h}\left(\left|c_{3} z^{2}+c_{4} z \bar{z}+c_{5} \bar{z}^{2}\right|^{2}\right)= & \Lambda_{4}^{h}\left(c_{3} \bar{c}_{3} z^{2} \bar{z}^{2}+c_{3} \bar{c}_{4} z^{3} \bar{z}+c_{3} \bar{c}_{5} z^{4}+c_{4} \bar{c}_{3} z \bar{z}^{3}+c_{4} \bar{c}_{4} z^{2} \bar{z}^{2}\right. \\
& \left.+c_{4} \bar{c}_{5} z^{3} \bar{z}+c_{5} \bar{c}_{3} \bar{z}^{4}+c_{5} \bar{c}_{4} z \bar{z}^{3}+c_{5} \bar{c}_{5} z^{2} \bar{z}^{2}\right) \\
= & \left(\left(\begin{array}{lll}
1 & b & 0 \\
b & 1 & b \\
0 & b & 1
\end{array}\right)\left(\begin{array}{l}
c_{3} \\
c_{4} \\
c_{5}
\end{array}\right)\left(\begin{array}{l}
c_{3} \\
c_{4} \\
c_{5}
\end{array}\right)\right) .
\end{aligned}
$$

The eigenvalues of this matrix are $1,1+\sqrt{2} b$ and $1-\sqrt{2} b$. For $\sqrt{2} / 2<b<1$, there is a negative eigenvalue, with eigenvector $(1,-\sqrt{2}, 1)$, which corresponds to the polynomial

$$
p(z, \bar{z}):=\left|z^{2}-\sqrt{2} z \bar{z}+\bar{z}^{2}\right|^{2} \in \mathcal{S}^{2} .
$$


However, the compressions of the matrix to $c_{4}=0$ and to $c_{5}=0$ are positive; therefore (by Step 2) $\left.\Lambda_{4}^{h}\right|_{\Gamma_{4}^{h}} \geq 0$, and of course $\left.\Lambda_{4}^{h}\right|_{\operatorname{int}\left(\Gamma_{4}^{h}\right)}>0$.

Summarizing, we have found $p \in \mathcal{S}^{2}$ such that $\Lambda_{4}^{h}(p)<0$ and $\left.\Lambda_{4}^{h}\right|_{\operatorname{int}\left(W_{4}^{h}\right)}>0$. In $E=\mathbb{R}[x, y]_{4}$ we set $K=\mathcal{W}_{4}, M=\operatorname{ker} \Lambda_{4}^{h}$ and then have

$$
M \cap \operatorname{int}(K)=\operatorname{ker} \Lambda_{4}^{h} \cap \operatorname{int}\left(\mathcal{W}_{4}\right)=\operatorname{ker} \Lambda_{4}^{h} \cap \operatorname{int}\left(\mathcal{W}_{4}^{h}\right)=\emptyset,
$$

and $\operatorname{int}(K)=\operatorname{int}\left(\mathcal{W}_{4}\right) \neq \emptyset$, so there exists $\Lambda_{4}$ on $\mathbb{R}[x, y]_{4}$ such that $\operatorname{ker} \Lambda_{4} \cap \operatorname{int}\left(\mathcal{W}_{4}\right)=\emptyset$. We now switch to $E=\mathbb{R}[x, y]_{5}$ and consider $K=\mathcal{W}_{5}$ and $M=\operatorname{ker} \Lambda_{4}$. Since

$$
\operatorname{ker} \Lambda_{4} \cap \operatorname{int}\left(\mathcal{W}_{5}\right)=\operatorname{ker} \Lambda_{4} \cap \operatorname{int}\left(\mathcal{W}_{4}\right)=\emptyset,
$$

and $\operatorname{int}\left(\mathcal{W}_{5}\right) \neq \emptyset$, we see that there exists $\Lambda_{5}$ on $\mathbb{R}[x, y]_{5}$ such that $\operatorname{ker} \Lambda_{5} \cap \operatorname{int}\left(\mathcal{W}_{5}\right)=\emptyset$. Next, we consider $E=\mathbb{R}[x, y]_{6}, K=\mathcal{W}_{6}$ and $M=\operatorname{ker} \Lambda_{5}$, and continue this process ad infinitum. The resulting linear functional $\Lambda$ has the right separation properties.

The solution of Problem 1.1 ([CuP2, Theorem 2.1]), establishing a separation between subnormality and polynomial hyponormality, gives rise to a number of open questions and provides a new viewpoint for subnormal and hyponormal operator theory. On one hand, one can now study the class of polynomially hyponormal operators on its own, and seek to extend well-known properties of subnormal operators, or try to find useful characterizations. On the other hand, even if one were to argue that the new class remains a bit artificial (mainly because no concrete nontrivial examples exist), it is clear that its study is relevant in gaining a complete understanding of the relationship between subnormality and hyponormality. Either way, a multitude of problems arise, some of which we proceed to list.

Problem 2.3. Find a concrete example of a polynomially hyponormal operator which is not 2-hyponormal.

Problem 2.4. Find a concrete example of a non-subnormal polynomially hyponormal weighted shift.

We notice that the separating linear functional constructed above cannot correspond to a weighted shift, since in this case $\Lambda\left(z^{i} \bar{z}^{j}\right)$ must equal $\left(T^{i} e_{0}, T^{j} e_{0}\right)$ and so it must be zero when $i \neq j$. We are thus naturally led to the following question.

Problem 2.5. Does there exist a polynomially hyponormal weighted shift that is not 2-hyponormal?

Problem 2.6. Do polynomially hyponormal operators have nontrivial invariant subspaces?

Problem 2.7. Is the principal function of a polynomially hyponormal operator with trace-class self-commutator integer-valued?

PRoBlem 2.8. Is there a model for polynomially hyponormal operators with finite-rank self-commutator?

3. Truncated moment problems. We begin this section by recalling a few facts about unilateral weighted shifts. 
Definition 3.1. A weighted shift $W_{\alpha}$ is said to be recursively generated if there exist $p \geq 1$ and $\varphi_{0}, \ldots, \varphi_{p-1} \in \mathbb{R}$ such that

$$
\gamma_{n+p}=\varphi_{0} \gamma_{n}+\ldots+\varphi_{p-1} \gamma_{n+p-1} \quad(\text { for all } n \geq 0),
$$

where

$$
\gamma_{0}:=1, \quad \gamma_{n}:=\alpha_{0}^{2} \ldots \alpha_{n-1}^{2} \quad(n \geq 1)
$$

THEOREM 3.2 (C. Berger [Con, III.8.16]). $W_{\alpha}$ is subnormal if and only if $\gamma_{n}=$ $\int t^{n} d \mu(t)$ for some probability measure $\mu$ with $\operatorname{supp} \mu \subseteq\left[0,\left\|W_{\alpha}\right\|^{2}\right]$.

Theorem $3.3([\mathrm{CuF} 1])$. Assume that $W_{\alpha}$ is subnormal. Then

$$
W_{\alpha} \text { is recursively generated } \Leftrightarrow \operatorname{supp} \mu \text { is finite. }
$$

To find the measure $\mu$, let $r$ be the minimum integer satisfying (3.1) and consider

$$
g(t):=t^{r}-\left(\varphi_{0}+\ldots+\varphi_{r-1} t^{r-1}\right)
$$

TheOREm 3.4 ([CuF1, Theorem 3.9(i)]). g has exactly $r$ zeros,

$$
0 \leq s_{0}<\ldots<s_{r-1}=\left\|W_{\alpha}\right\|^{2} .
$$

Let

$$
\mu:=\rho_{0} \delta_{s_{0}}+\ldots+\rho_{r-1} \delta_{s_{r-1}}, \quad \text { where } \quad\left(\rho_{0} \rho_{1} \ldots \rho_{r-1}\right)^{T}:=V^{-1}\left(\gamma_{0} \gamma_{1} \ldots \gamma_{r-1}\right)^{T} .
$$

In the process of finding the measure $\mu$, we solved in [CuF1] the truncated Stieltjes moment problem; incidentally, it turned out that the measure we obtained there is as economical as possible (cf. [CuF1, Theorem 3.9]). We shall indicate below how a similar procedure will allow us to deal with other truncated moment problems on the real line and on the unit circle. For ease of explanation, we first focus on the case $m=2 k$ of the Stieltjes problem ( $\operatorname{supp} \mu \subseteq[0,+\infty)$ ). We begin by introducing some additional terminology.

Definition 3.5. For $k \geq 0$ and $\gamma \equiv\left(\gamma_{0}, \ldots, \gamma_{2 k}\right) \in \mathbb{R}^{2 k+1}$ given, with $\gamma_{0} \neq 0$, let

$$
A \equiv A(k):=\left(\gamma_{i+j}\right)_{i, j=0}^{k}=\left(\begin{array}{cccc}
\gamma_{0} & \gamma_{1} & \cdots & \gamma_{k} \\
\gamma_{1} & \gamma_{2} & \cdots & \gamma_{k+1} \\
\ldots \ldots & \ldots & \ldots & \ldots \\
\gamma_{k} & \gamma_{k+1} & \cdots & \gamma_{2 k}
\end{array}\right)
$$

and

$$
\mathbf{v}_{j}:=\left(\gamma_{j+l}\right)_{l=0}^{k}=\left(\begin{array}{c}
\gamma_{j} \\
\gamma_{j+1} \\
\cdots \\
\gamma_{j+k}
\end{array}\right) \quad(0 \leq j \leq k) .
$$

The (Hankel) rank of $\gamma, \operatorname{rank} \gamma$, is defined as follows: (i) if $A$ is nonsingular, $\operatorname{rank} \gamma:=$ $k+1$; (ii) if $A$ is singular, $\operatorname{rank} \gamma$ is the smallest integer $r$ such that $\mathbf{v}_{r} \in\left\langle\mathbf{v}_{0}, \ldots, \mathbf{v}_{r-1}\right\rangle$, that is, there exists a unique $r$-tuple $\varphi \equiv \varphi(\gamma)=\left(\varphi_{0}, \ldots, \varphi_{r-1}\right) \in \mathbb{R}^{r}$ such that $\mathbf{v}_{r}=$ $\varphi_{0} \mathbf{v}_{0}+\ldots+\varphi_{r-1} \mathbf{v}_{r-1}$.

The following result, based on J. L. Smul'jan's criterion for positivity of $2 \times 2$ block matrices [Smu], is a restatement of the structure theorem for positive Hankel matrices (Theorem 1.5). 
Theorem 3.6 ([CuF3, Theorem 2.4]). Let $\gamma \equiv\left(\gamma_{0}, \ldots, \gamma_{2 k}\right), \gamma_{2 k+1}, \gamma_{2 k+2}$ be given, and let

$$
\widetilde{A} \equiv A(k+1)=\left(\begin{array}{ccc} 
& & \\
& A & \left(\begin{array}{c}
\gamma_{k+1} \\
\ldots \\
\gamma_{2 k+1}
\end{array}\right) \\
\left(\gamma_{k+1}\right. & \ldots & \gamma_{2 k+1}
\end{array}\right) .
$$

Assume that $\widetilde{A} \geq 0$ and let $r:=\operatorname{rank} \gamma$. Then

(i) $A(r-1)$ is positive and invertible,

(ii)

$$
\left\{\begin{array}{l}
\gamma_{r}=\varphi_{0} \gamma_{0}+\ldots+\varphi_{r-1} \gamma_{r-1} \\
\gamma_{r+1}=\varphi_{0} \gamma_{1}+\ldots+\varphi_{r-1} \gamma_{r} \\
\ldots \ldots \ldots \ldots \ldots \ldots \ldots \ldots \ldots \\
\gamma_{2 k}=\varphi_{0} \gamma_{2 k-r}+\ldots+\varphi_{r-1} \gamma_{2 k-1} \\
\gamma_{2 k+1}=\varphi_{0} \gamma_{2 k+1-r}+\ldots+\varphi_{r-1} \gamma_{2 k}
\end{array}\right.
$$

(iii) $\gamma_{2 k+2} \geq \varphi_{0} \gamma_{2 k+2-r}+\ldots+\varphi_{r-1} \gamma_{2 k+1}$.

Corollary 3.7 ([CuF3, Corollary 2.5]). Suppose $\widetilde{A} \geq 0$ and let $r:=\operatorname{rank} \gamma$.

(i) $\operatorname{rank} A=r$;

(ii) $r \leq \operatorname{rank} \widetilde{A} \leq r+1$; moreover,

$$
\operatorname{rank} \widetilde{A}=r+1 \Leftrightarrow \gamma_{2 k+2}>\varphi_{0} \gamma_{2 k+2-r}+\cdots+\varphi_{r-1} \gamma_{2 k+1} .
$$

Theorem 3.8 (Extension Principle [CuF3, Theorem 2.6]). Let $A=A(k)$ and let $r:=\operatorname{rank} \gamma$. If $A \geq 0$, then the following are equivalent:

(i) A has a positive Hankel extension;

(ii) $\operatorname{rank} A=r$;

(iii) There exists $\varphi_{0}, \ldots, \varphi_{r-1} \in \mathbb{R}$ such that

$$
\gamma_{j}=\varphi_{0} \gamma_{j-r}+\ldots+\varphi_{r-1} \gamma_{j-1} \quad(r \leq j \leq 2 k) .
$$

Moreover, if (i) holds and $r \leq k$, then in any positive Hankel extension of $A \gamma_{2 k+1}$ is recursively determined by $\varphi(\gamma)$, i.e.,

$$
\gamma_{2 k+1}=\varphi_{0} \gamma_{2 k+1-r}+\ldots+\varphi_{r-1} \gamma_{2 k} .
$$

Re mark 3.9. Assume $A(k)$ has a positive Hankel extension. Then if $A$ is invertible $\gamma_{2 k+1}$ can be chosen arbitrarily, while if $A$ is singular $\gamma_{2 k+1}$ is uniquely determined. In either case, once $\gamma_{2 k+1}$ is prescribed, $\gamma_{2 k+2}$ can be chosen as any value satisfying condition (iii) of Theorem 3.6.

With these preliminaries, we can now give an elementary algebraic treatment of the truncated moment problem for measures with support in the real line. For $\widetilde{\gamma} \equiv$ $\left(\gamma_{0}, \ldots, \gamma_{2 k}, \gamma_{2 k+1}\right)$

$$
g_{\gamma}(t):=t^{r}-\left(\varphi_{0}+\ldots+\varphi_{r-1} t^{r-1}\right) \quad(t \in \mathbb{R})
$$

is the generating function of $\widetilde{\gamma}$. We start with the basic case.

\subsection{The truncated Hamburger moment problem}

Theorem 3.10 (Existence Theorem; odd case [CuF3, Theorem 3.1]). Let $\widetilde{\gamma} \equiv\left(\gamma_{0}, \ldots\right.$ $\left.\ldots, \gamma_{2 k}, \gamma_{2 k+1}\right), \gamma_{0} \neq 0$. The following are equivalent: 
(i) There exists a positive Borel measure $\mu$ on $\mathbb{R}$ such that

$$
\gamma_{j}=\int t^{j} d \mu(t) \quad(0 \leq j \leq 2 k+1)
$$

(ii) There exists a compactly supported representing measure for $\widetilde{\gamma}$;

(iii) There exists a finitely atomic representing measure for $\widetilde{\gamma}$;

(iv) There exists an $r$-atomic representing measure for $\widetilde{\gamma}$, whose support consists of the roots of $g_{\tilde{\gamma}}$;

(v) $A \equiv A(k) \geq 0$ and $\mathbf{v}_{k+1} \in \operatorname{Ran} A$;

(vi) $A(k+1) \geq 0$ for some choice of $\gamma_{2 k+2} \in \mathbb{R}$.

Theorem 3.10 reduces the question of existence of representing measures to two basic problems, which can be approached by means of symbolic manipulation:

(a) When is $\mathbf{v}_{k+1} \in \operatorname{Ran} A(k)$ ?

(b) When is $\sigma(A(k)) \subseteq[0,+\infty)$ ?

For $x_{0}, \ldots, x_{p} \in \mathbb{R}$, the Vandermonde matrix associated with $x:=\left(x_{0}, \ldots, x_{p}\right)$ is defined as

$$
V_{x}:=\left(\begin{array}{cccc}
1 & 1 & \ldots & 1 \\
x_{0} & x_{1} & \ldots & x_{p} \\
\ldots \ldots \ldots \ldots \ldots & \ldots \\
x_{0}^{p} & x_{1}^{p} & \ldots & x_{p}^{p}
\end{array}\right)
$$

it is well known that $V_{x}$ is invertible if and only if the $x_{i}$ 's are all distinct. The following results give a recipe for constructing an interpolating measure.

Proposition 3.11 ([CuF3, Proposition 3.3]). If $A \equiv A(k)$ is positive and invertible, then $g_{\tilde{\gamma}}$ has $k+1$ distinct real roots, $x_{0}, \ldots, x_{k}$. Thus $V_{x}$ is invertible, and if $\widehat{\rho}:=V_{x}^{-1} \mathbf{v}_{0}$, then $\rho_{j}>0 \quad(0 \leq j \leq k)$. Moreover, if $\mu:=\sum_{i=0}^{k} \rho_{i} \delta_{x_{i}}$, then $\gamma_{j}=\int t^{j} d \mu(t)(0 \leq j \leq$ $2 k+1)$.

Corollary 3.12 ([CuF3, Corollary 3.4]). Assume that $A \equiv A(k) \geq 0$ and that $\mathbf{v}_{k+1} \in$ $\operatorname{Ran} A$. Then there exists a measure $\mu$, with supp $\mu=\mathcal{Z}\left(g_{\tilde{\gamma}}\right)$ (the zero set of $\left.g_{\tilde{\gamma}}\right)$ such that

$$
\gamma_{j}=\int t^{j} d \mu \quad(0 \leq j \leq 2 k+1)
$$

Theorem 3.13 (Uniqueness Theorem; odd case [CuF3, Theorem 3.8]). Let $\widetilde{\gamma}=\left(\gamma_{0}\right.$, $\left.\ldots, \gamma_{2 k+1}\right), \gamma_{0} \neq 0$, and suppose $\widetilde{\gamma}$ has a representing measure, i.e., $A(k) \geq 0$ and $\mathbf{v}_{k+1} \in \operatorname{Ran} A(k)$.

(i) If $r \leq k$, then the truncated moment problem has the unique solution

$$
\mu=\sum_{i=0}^{k} \rho_{i} \delta_{x_{i}},
$$

where $\operatorname{supp} \mu=\left\{x_{0}, \ldots, x_{r-1}\right\}=\mathcal{Z}\left(g_{\tilde{\gamma}}\right)$ and $\widehat{\rho}$ is given by $\widehat{\rho}=V_{x}^{-1} \mathbf{v}_{0}$, with $x:=$ $\left(x_{0}, \ldots, x_{r-1}\right)$.

(ii) If $r=k+1$, then the truncated moment problem has infinitely many solutions.

3.2. The truncated Hausdorff moment problem. This moment problem requires that the measure $\mu$ have support contained in a prescribed interval $[a, b]$. For simplicity, we 
restrict attention to the case $m=2 k$, and we only mention the existence theorem; complete details can be found in [CuF3].

Theorem 3.14 (Existence; odd case [CuF3, Theorem 4.1]). Let $\widetilde{\gamma}=\left(\gamma_{0}, \ldots, \gamma_{2 k+1}\right)$, $\gamma_{0}>0$. The following are equivalent:

(i) There exists a positive Borel measure $\mu$ satisfying (3.2) and $\operatorname{supp} \mu \subseteq[a, b]$;

(ii) There exists a finitely atomic measure $\mu$ satisfying (3.2) and $\operatorname{supp} \mu \subseteq[a, b]$;

(iii) There exists an r-atomic measure $\mu$ satisfying (3.2) and $\operatorname{supp} \mu=\mathcal{Z}\left(g_{\tilde{\gamma}}\right)$;

(iv) $A(k) \geq 0, \mathbf{v}_{k+1} \in \operatorname{Ran} A(k)$, and $b A(k) \geq B(k) \geq a A(k)$.

3.3. The truncated Stieltjes moment problem. Here we require that the support be contained in the nonnegative $x$-axis; the following result is easily obtained from Theorem 3.14 , by letting $a=0$ and $b \rightarrow+\infty$.

Theorem 3.15 (Existence; odd case $\left[\mathrm{CuF} 3\right.$, Theorem 5.1]). Let $\widetilde{\gamma}=\left(\gamma_{0}, \ldots, \gamma_{2 k+1}\right)$, $\gamma>0$. The following are equivalent:

(i) There exists a positive Borel measure $\mu$ satisfying (3.2) and $\operatorname{supp} \mu \subseteq[0,+\infty)$;

(ii) There exists a finitely atomic measure $\mu$ satisfying (3.2) and supp $\mu \subseteq[0,+\infty)$;

(iii) There exists an $r$-atomic measure $\mu$ satisfying (3.2) and $\operatorname{supp} \mu=\mathcal{Z}\left(g_{\tilde{\gamma}}\right)$;

(iv) $A(k) \geq 0, B(k) \geq 0$ and $\mathbf{v}(k+1, k) \in \operatorname{Ran} A(k)$.

3.4. The truncated Toeplitz moment problem

Definition 3.16. Given $\gamma \equiv\left(\gamma_{-k}, \ldots, \gamma_{0}, \ldots, \gamma_{k}\right), \gamma_{-j}=\bar{\gamma}_{j}(j=1, \ldots, k), \gamma_{0}>0$ we let

$$
T_{\gamma} \equiv T(k):=\left(\begin{array}{ccccccc}
\gamma_{0} & \gamma_{1} & \ldots & \gamma_{i-1} & \gamma_{i} & \ldots & \gamma_{k} \\
\gamma_{-1} & \gamma_{0} & \ldots & \gamma_{i-2} & \gamma_{i-1} & \ldots & \gamma_{k-1} \\
\ldots \ldots & \ldots \ldots \ldots \ldots \ldots \ldots \ldots \ldots \ldots \ldots \ldots \ldots \ldots \ldots \ldots \ldots \\
\gamma_{1-i} & \gamma_{2-i} & \ldots & \gamma_{0} & \gamma_{1} & \ldots & \gamma_{k+1-i} \\
\gamma_{-i} & \gamma_{1-i} & \ldots & \gamma_{-1} & \gamma_{0} & \ldots & \gamma_{k-i} \\
\ldots & \ldots & \ldots & \ldots & \ldots & \ldots & \ldots \\
\gamma_{-k} & \gamma_{1-k} & \ldots & \gamma_{-k+i-1} & \gamma_{-k+i} & \ldots & \gamma_{0}
\end{array}\right)
$$

denote the associated Toeplitz matrix. The (Toeplitz) rank of $\gamma, \operatorname{rank} \gamma$, is defined as follows:

(i) If $T(k)$ is invertible, $r=k+1$;

(ii) If $T(k)$ singular, rank $\gamma$ is the smallest integer such that $\mathbf{v}_{r} \in\left\langle\mathbf{v}_{0}, \ldots, \mathbf{v}_{r-1}\right\rangle$ (and in this case, we let $\varphi_{\gamma} \equiv\left(\varphi_{0}, \ldots, \varphi_{r-1}\right)$ denote the unique collection of scalars such that $\left.\mathbf{v}_{r}=\varphi_{0} \mathbf{v}_{0}+\ldots+\varphi_{r-1} \mathbf{v}_{r-1}\right)$.

Proposition 3.17 ([CuF3, Proposition 6.3]). Let $r:=\operatorname{rank} \gamma$. If $T(k) \geq 0$, then $T(r-1)$ is nonsingular.

The following is the analogue of Theorem 1.5 for positive Toeplitz matrices; note that unlike the situation for Hankel matrices, in the singular case all entries are fully determined.

TheOREM 3.18 (Structure Theorem for positive Toeplitz matrices [CuF3, Theorem 6.4]). If $T(k)$ is singular and positive, then 
(i) $T(r-1)$ is positive and invertible; and

(ii) $\varphi_{\gamma}$ satisfies

$$
\gamma_{j}=\varphi_{0} \gamma_{j-r}+\ldots+\varphi_{r-1} \gamma_{r-1}, \quad r-k \leq j \leq k .
$$

Conversely, if there exists $i, 1 \leq i \leq k$, and scalars $\varphi_{0}, \ldots, \varphi_{i-1}$ such that $T(i-1) \geq 0$ and (ii) holds, then $T(k)$ is positive.

Corollary 3.19 ([CuF3, Corollary 6.6]). If $T_{\gamma} \geq 0$ is singular, then it has a unique positive Toeplitz extension.

Proposition 3.20 ([CuF3, Proposition 6.8 and Corollary 6.9]). If $T_{\gamma}$ is positive and invertible, then $T_{\gamma}$ has infinitely many positive invertible Toeplitz extensions.

The next result establishes necessary and sufficient conditions for the existence of representing measures.

TheOREM 3.21 ([CuF3, Theorem 6.12]). Let $\gamma=\left(\gamma_{-k}, \ldots, \gamma_{0}, \ldots \gamma_{k}\right) \in \mathbb{C}^{2 k+1}, \gamma_{0}>0$, $\gamma_{-j}=\bar{\gamma}_{j}$ be given. Then there exists a representing measure $\mu$ for $\gamma$ if and only if $T_{\gamma} \geq 0$. In this case, $\mu$ can be chosen to have exactly $r$ atoms.

As for uniqueness, an application of the F. and M. Riesz Theorem leads to a proof of uniqueness for the truncated Toeplitz moment problem whenever $T_{\gamma}$ is singular [CuF3, Remark 6.13]; the invertible case, however, allows for infinitely many solutions.

4. The truncated complex moment problem. Given $\gamma: \gamma_{00}, \gamma_{01}, \gamma_{10}, \ldots$ $\ldots, \gamma_{0,2 n}, \ldots, \gamma_{2 n, 0}$, with $\gamma_{00}>0$ and $\gamma_{j i}=\bar{\gamma}_{i j}$, the truncated complex moment problem (TCMP) entails finding a positive Borel measure $\mu$ supported in the complex plane $\mathbb{C}$ such that

$$
\gamma_{i j}=\int \bar{z}^{i} z^{j} d \mu \quad(0 \leq i+j \leq 2 n)
$$

$\mu$ is called a representing measure for $\gamma$. Naturally associated with each TCMP there is a moment matrix $M(n)$, whose construction we will consider a bit later.

In $[\mathrm{CuF} 4]$, we have introduced an approach based on matrix positivity and extension, combined with a new "functional calculus" for the columns of $M(n)$ (to be labelled $1, Z, \bar{Z}, Z^{2}, \bar{Z} Z, \bar{Z}^{2}, \ldots$, etc.), which has allowed us to establish that a solution always exists in the following three main cases: (i) the TCMP is of flat data type (this subsumes all previous results for the Hamburger, Stieltjes, Hausdorff and Toeplitz problems discussed in Section 3; (ii) $n=1$ (this is the quadratic moment problem); (iii) the column $\bar{Z}$ is a linear combination of 1 and $Z$, that is, $\bar{Z}=\alpha 1+\beta Z$ for some $\alpha, \beta \in \mathbb{C}$; and (iv) the analytic column $Z^{k}$ is a linear combination of columns corresponding to monomials of lower degree, for some $k \leq[n / 2]+1$, that is, $Z^{k} \in\left\langle\bar{Z}^{i} Z^{j}\right\rangle_{0 \leq i+j \leq k-1}$.

Our techniques permit us to deal at the same time with truncated moment problems in one or several real or complex variables, and to give concrete algorithms to provide finitelyatomic representing measures whose atoms and densities can be explicitly computed.

4.1. Basic positivity condition. As in the case of measures supported in the real line, a calculation involving polynomials can be exploited to derive the following necessary positivity condition. First, we need some terminology. We shall let $\mathcal{P}_{n}$ denote the finite 
dimensional space consisting of all complex polynomials in $z$ and $\bar{z}$ of total degree at most $n$. Clearly, $\operatorname{dim} \mathcal{P}_{n}=m:=(n+1)(n+2) / 2$. For $p \in \mathcal{P}_{n}, p(z, \bar{z}) \equiv \sum_{0 \leq i+j \leq n} a_{i j} \bar{z}^{i} z^{j}$, and if we assume that a positive Borel measure satisfies (4.1), then

$$
0 \leq \int|p(z, \bar{z})|^{2} d \mu(z, \bar{z})=\sum_{i j k \ell} a_{i j} \bar{a}_{k \ell} \int \bar{z}^{i+\ell} z^{j+k} d \mu(z, \bar{z})=\sum_{i j k \ell} a_{i j} \bar{a}_{k \ell} \gamma_{i+\ell, j+k} .
$$

To understand this notion of positivity, we first define the block $M[i, j]$ of size $(i+1) \times$ $(j+1)$, as follows:

$$
M[i, j]:=\left(\begin{array}{cccc}
\gamma_{i, j} & \gamma_{i+1, j-1} & \ldots & \gamma_{i+j, 0} \\
\gamma_{i-1, j+1} & \gamma_{i, j} & \ldots & \gamma_{i+j-1,1} \\
\ldots \ldots \ldots & \ldots \ldots \ldots \ldots \ldots \ldots \ldots \\
\gamma_{0, i+j} & \gamma_{1, i+j-1} & \ldots & \gamma_{i, j}
\end{array}\right) \quad(0 \leq i, j \leq n) ;
$$

for instance,

$$
M[3,2]:=\left(\begin{array}{lll}
\gamma_{32} & \gamma_{41} & \gamma_{50} \\
\gamma_{23} & \gamma_{32} & \gamma_{41} \\
\gamma_{14} & \gamma_{23} & \gamma_{32} \\
\gamma_{05} & \gamma_{14} & \gamma_{23}
\end{array}\right) .
$$

$M(n)$ is then the block matrix given as $(M[i, j])_{i, j=0}^{n}$, for example,

$$
M(1)=\left(\begin{array}{lll}
\gamma_{00} & \gamma_{01} & \gamma_{10} \\
\gamma_{10} & \gamma_{11} & \gamma_{20} \\
\gamma_{01} & \gamma_{02} & \gamma_{11}
\end{array}\right)
$$

and

$$
M(2)=\left(\begin{array}{llllll}
\gamma_{00} & \gamma_{01} & \gamma_{10} & \gamma_{02} & \gamma_{11} & \gamma_{20} \\
\gamma_{10} & \gamma_{11} & \gamma_{20} & \gamma_{12} & \gamma_{21} & \gamma_{30} \\
\gamma_{01} & \gamma_{02} & \gamma_{11} & \gamma_{03} & \gamma_{12} & \gamma_{21} \\
\gamma_{20} & \gamma_{21} & \gamma_{12} & \gamma_{22} & \gamma_{31} & \gamma_{40} \\
\gamma_{11} & \gamma_{12} & \gamma_{21} & \gamma_{13} & \gamma_{22} & \gamma_{31} \\
\gamma_{02} & \gamma_{03} & \gamma_{12} & \gamma_{04} & \gamma_{13} & \gamma_{22}
\end{array}\right)
$$

The positivity in (4.2) can now be expressed succinctly as the positivity of $M(n)$, that is,

$$
\sum_{i j k \ell} a_{i j} \bar{a}_{k \ell} \gamma_{i+\ell, j+k} \geq 0 \Leftrightarrow M(n) \geq 0 \quad([\mathrm{CuF} 4, \text { Chapter 3, (3.2)]; cf. [StSz4] }) .
$$

This positivity condition is by no means sufficient. For example, there exist $\gamma_{00}, \gamma_{01}$, $\gamma_{10}, \ldots, \gamma_{06}, \ldots, \gamma_{60}$ with positive invertible moment matrix $M(3)$ but admitting no representing measure (see Section 8 below).

4.2. Functional calculus. In analogy with our discussion of Hankel matrices preceding Theorem 1.5, we introduce the following lexicographic order on the rows and columns of $M(n): 1, Z, \bar{Z}, Z^{2}, \bar{Z} Z, \bar{Z}^{2}, Z^{3}, \bar{Z} Z^{2}, \bar{Z}^{2} Z, \bar{Z}^{3}, \ldots, Z^{n}, \bar{Z} Z^{n-1}, \ldots, \bar{Z}^{n-1} Z, \bar{Z}^{n}$. For $p \in$ $\mathcal{P}_{n}, p(z, \bar{z}) \equiv \sum_{0 \leq i+j \leq n} a_{i j} \bar{z}^{i} z^{j}$ define $p(Z, \bar{Z}):=\sum a_{i j} \bar{Z}^{i} Z^{j}$. Recall that Theorem 1.5 states that if we label the columns of a positive singular Hankel matrix $H(k) \equiv\left(h_{i+j}\right)_{i, j=0}^{k}$ as $1, T, \ldots, T^{k}$, and if $T^{r}=\varphi_{0} 1+\ldots+\varphi_{r-1} T^{r-1}$ for some scalars $\varphi_{0}, \ldots, \varphi_{r-1}$, then $\left.T^{r+s}=\varphi_{0} T^{s}+\ldots+\varphi_{r-1} T^{r+s-1}(0 \leq s \leq k-r)\right)$, a property we called recursiveness. 
The support of any representing measure must then lie in the solution set of the equation

$$
g(t):=t^{r}-\left(\varphi_{0}+\ldots+\varphi_{r-1} t^{r-1}\right)=0 .
$$

The following is our analogue of recursiveness for the TCMP:

(RG) If $p, q, p q \in \mathcal{P}_{n}$, and $p(Z, \bar{Z})=\mathbf{0}$, then $(p q)(Z, \bar{Z})=\mathbf{0}$,

a property obviously necessary for the existence of a representing measure. We thus reach the following conjecture.

CONJECTURE 4.1. Let $\gamma$ be a truncated moment sequence. The following assertions are equivalent.

(i) $\gamma$ has a representing measure;

(ii) $\gamma$ has a representing measure with moments of all orders;

(iii) $\gamma$ has a compactly supported representing measure;

(iv) $\gamma$ has a finitely atomic representing measure;

(v) $\gamma$ has a rank $M(n)$-atomic representing measure;

(vi) $M(n) \geq 0$ and $M(n)$ admits a flat (i.e., rank-preserving) extension $M(n+1)$.

It is clear that (iv) $\Rightarrow($ iii $) \Rightarrow($ ii $) \Rightarrow(\mathrm{i})$. In recent work $[\mathrm{CuF} 6]$, we have adapted results of V. Tchakaloff [Tch] and I. P. Mysovskikh [Mys] to prove (i) $\Rightarrow(i v)$; thus, conditions (i), (ii), (iii) and (iv) are all equivalent. Independently, M. Putinar [Pu6] has found a different proof of $(\mathrm{i}) \Rightarrow(\mathrm{iv})$, also based on extending results of [Tch]. (Somewhat earlier, we had obtained (iii) $\Rightarrow$ (iv) by adapting [Tch], and J. McCarthy had communicated to us another proof of the same implication, using convexity theory.)

Theorem 6.4 below shows that (v) and (vi) are equivalent, and clearly (v) $\Rightarrow(\mathrm{iv})$; however, J. McCarthy $[\mathrm{McC}]$ has recently proved that there exist truncated moment sequences $\gamma$ having representing measures, but such that $M(n)(\gamma)$ does not have a flat extension $M(n+1)$. Thus $(\mathrm{i}) \nRightarrow(\mathrm{v})$ and Conjecture 4.1 is false as stated. McCarthy's dimension-theoretic result actually shows that moment sequences $\gamma$ admitting no flat extensions are in a sense generic: among moment sequences $\gamma$ with representing measures, those with rank $M(n)(\gamma)$-atomic representing measures are rare. On the other hand, it follows from the equivalence of (i) and (iv) and from the equivalence of (v) and (vi) that a truncated moment sequence $\gamma$ has a representing measure if and only if for some $k \geq 0, M(n)(\gamma)$ admits a positive extension $M(n+k)$ which in turn has a flat extension $M(n+k+1)$.

We shall discuss the above implications, along with the main results in $[\mathrm{CuF} 4]$ and $[\mathrm{CuF} 5]$, in the next several sections.

5. Finite-rank infinite moment matrices. For the truncated Hamburger moment problem, the concrete construction of a representing measure described in Section 3 admits an alternative approach, more abstract, but perhaps quite revealing in terms of providing further insight into the problem. That is, recall that our basic ploy was to extend the given $(k+1) \times(k+1)$ positive Hankel matrix, $A(k)$, to a $(k+2) \times(k+2)$ Hankel matrix $A(k+1)$, which was still positive, and this because we insisted on preserving the matrix rank, i.e., $\operatorname{rank} A(k)=\operatorname{rank} A(k+1)$. Once one Hankel extension has been 
obtained, we can iterate the process to produce a second Hankel extension, $A(k+2)$, still of equal rank (hence positive), and so on ad infinitum. In the end, we obtain an infinite Hankel matrix whose rank equals that of the original matrix $A(k)$. Since it is well known that such matrices are associated with positive Borel measures, the existence of representing measures for the truncated Hamburger moment problem (and a fortiori for the Hamburger and Hausdorff problems) follows.

In this section we establish the existence of representing measures for finite-rank infinite moment matrices, with an eye towards using this result later, in a manner resembling the situation described in the previous paragraph. First, it is necessary to give a characterization of the condition of being a moment matrix, that is, one built according to the recipe in (4.3).

Definition 5.1. For $p \in \mathcal{P}_{n}, p(z, \bar{z}) \equiv \sum a_{i j} \sum a_{i j} \bar{z}^{i} z^{j}$, let

$$
\widehat{p}:=\sum a_{i j} e_{i j} \in \mathbb{C}^{m} \text {, }
$$

and for $p, q \in \mathcal{P}_{n}$ let

$$
\langle p, q\rangle_{A}:=\langle A \widehat{p}, \widehat{q}\rangle \quad\left(p, q \in \mathcal{P}_{n}\right)
$$

In particular,

$$
\left\langle\bar{z}^{i} z^{j}, \bar{z}^{k} z^{\ell}\right\rangle_{A}=\left\langle A e_{i j}, e_{k \ell}\right\rangle=A_{(k, \ell)(i, j)} .
$$

If $A$ is self-adjoint, $\langle\cdot, \cdot\rangle_{A}$ is hermitian.

Theorem 5.2 (intrinsic characterization of moment matrices [CuF4, Theorem 2.1]). Let $n \geq 0$ and let $A$ be given. There exists a truncated moment sequence $\gamma \equiv$ $\left(\gamma_{i j}\right)_{0 \leq i+j \leq 2 n}, \gamma_{i j}=\bar{\gamma}_{j i}, \gamma_{00}>0$, such that $A=M(n)(\gamma)$ if and only if

(0) $\langle 1,1\rangle_{A}>0$;

(1) $A=A^{*}$;

(2) $\langle p, q\rangle_{A}=\langle\bar{q}, \bar{p}\rangle_{A}\left(p, q \in \mathcal{P}_{n}\right)$ (symmetry);

(3) $\langle z p, q\rangle_{A}=\langle p, \bar{z} q\rangle\left(p, q \in \mathcal{P}_{n-1}\right)$ (Hankel-type property);

(4) $\langle z p, z q\rangle_{A}=\langle\bar{z} p, \bar{z} q\rangle_{A}\left(p, q \in \mathcal{P}_{n-1}\right)$ (normality).

Our next result is the analog for moment matrices of Theorem 1.5.

Theorem 5.3 ([CuF4, Theorem 3.14]). Let $M(n) \geq 0$. If $f, g, f g \in \mathcal{P}_{n-1}$ and $f(Z, \bar{Z})$ $=0$, then $(f g)(Z, \bar{Z})=0$.

In order to both locate the support of a candidate for representing measure and to build finitely atomic measures $\mu$ associated with finite-rank infinite positive moment matrices, we studied in $[\mathrm{CuF} 4$, Chapter 4] the linear map from the space of polynomials in $z$ and $\bar{z}$ to the column space of an infinite moment matrix $M, \varphi: \mathbb{C}[z, \bar{z}] \rightarrow \mathcal{C}_{M}$, given by $\varphi\left(\bar{z}^{i} z^{j}\right):=\bar{Z}^{i} Z^{j}, i, j \geq 0$. Clearly

$$
\varphi(p)=M \widehat{p}=p(Z, \bar{Z})=\sum a_{i j} \bar{Z}^{i} Z^{j} .
$$

Let

$$
\mathcal{N}:=\{p \in \mathbb{C}[z, \bar{z}]:\langle M \widehat{p}, \widehat{p}\rangle=0\} \quad \text { and } \quad \operatorname{ker} \varphi:=\{p \in \mathbb{C}[z, \bar{z}]: \varphi(p)=\mathbf{0}\} .
$$

It is easy to see that $\operatorname{ker} \varphi \subseteq \mathcal{N}$. The next lemma establishes the opposite containment. 
Lemma 5.4 ([CuF4, Lemma 4.2]). Let $M$ be a positive infinite matrix. Then $\mathcal{N}=\operatorname{ker} \varphi$.

If we consider the quotient space $\mathbb{C}[z, \bar{z}] / \mathcal{N}$, and if we recall that $\langle p, q\rangle_{M}:=\langle M \hat{p}, \widehat{q}\rangle$ $(p, q \in \mathbb{C}[z, \bar{z}])$, we can see that $\mathbb{C}[z, \bar{z}] / \mathcal{N}$ admits a pre-Hilbert space structure, given by $\langle f+\mathcal{N}, g+\mathcal{N}\rangle(f, g \in \mathbb{C}[z, \bar{z}])$. The next result guarantees that the operator of multiplication by $z, M_{z}$, factors through $\mathcal{N}$, giving rise to an induced multiplication on $\mathbb{C}[z, \bar{z}] / \mathcal{N}$, which we will still denote by $M_{z}$.

Proposition 5.5 ([CuF4, Proposition 4.3]). $\operatorname{ker} \varphi$ is an ideal of $\mathbb{C}[z, \bar{z}]$.

LEMMA 5.6 ([CuF4, Lemma 4.4]). Let $M$ be a finite-rank positive infinite moment matrix. Then $\mathbb{C}[z, \bar{z}] / \mathcal{N}$ is a finite dimensional Hilbert space and $\operatorname{dim} \mathbb{C}[z, \bar{z}] / \mathcal{N}=\operatorname{rank} M$.

Proof. Consider

$$
\Phi: \mathcal{C}_{M} \rightarrow \mathbb{C}[z, \bar{z}] / \mathcal{N}
$$

defined by

$$
\Phi(p(Z, \bar{Z})):=p+\mathcal{N}, \quad p \in \mathbb{C}[z, \bar{z}] .
$$

If $q \in \mathbb{C}[z, \bar{z}]$ and $q(Z, \bar{Z})=p(Z, \bar{Z})$, then $q-p \in \operatorname{ker} \varphi=\mathcal{N}$, so $q+\mathcal{N}=p+\mathcal{N}$. Thus, $\Phi$ is well-defined, linear, and surjective. If $\Phi(p(Z, \bar{Z}))=0$, then $p \in \mathcal{N}=\operatorname{ker} \varphi$, so $p(Z, \bar{Z})=$ $\varphi(p)=0$. Thus $\Phi$ is an isomorphism, so $\operatorname{dim} \mathbb{C}[z, \bar{z}] / \mathcal{N}=\operatorname{dim} \mathcal{C}_{M}=\operatorname{rank} M<\infty$, and therefore $\mathbb{C}[z, \bar{z}] / \mathcal{N}$ is a complete pre-Hilbert space.

LEMma 5.7 ([CuF4, Lemma 4.5]). Let $M$ be a finite-rank positive infinite moment matrix. Then $M_{z}$, acting on $\mathbb{C}[z, \bar{z}] / \mathcal{N}$, is normal.

The next result allows us to "count" the number of atoms in a representing measure.

Proposition 5.8 ([CuF4, Proposition 4.6]). Let $M$ be an infinite moment matrix with representing measure $\mu$. Then card $\operatorname{supp} \mu=\operatorname{rank} M$.

We are now ready to present our existence and uniqueness result for finite-rank infinite moment matrices.

TheOrem 5.9 ([CuF4, Theorem 4.7]). Let $M$ be a finite-rank positive infinite moment matrix. Then $M$ has a unique representing measure, which is $\operatorname{rank} M$-atomic. In this case, let $r:=\operatorname{rank} M$; there exist unique scalars $\alpha_{0}, \ldots, \alpha_{r-1}$ such that $Z^{r}=\alpha_{0} 1+\ldots+$ $\alpha_{r-1} Z^{r-1}$. The unique representing measure for $M$ has support equal to the $r$ distinct roots $z_{0}, \ldots, z_{r-1}$ of the polynomial $z^{r}-\left(\alpha_{0}+\ldots+\alpha_{r-1} z^{r-1}\right)$, and densities $\rho_{0}, \ldots, \rho_{r-1}$ determined by the Vandermonde equation

$$
V_{z}\left(\begin{array}{llll}
\rho_{0} & \rho_{1} & \ldots & \rho_{r-1}
\end{array}\right)^{T}=\left(\begin{array}{llll}
\gamma_{00} & \gamma_{01} & \ldots & \gamma_{0, r-1}
\end{array}\right)^{T},
$$

where $z:=\left(z_{0}, \ldots, z_{r-1}\right)$.

Proof. $M_{z}$, acting on $\mathbb{C}[z, \bar{z}] / \mathcal{N}$, is normal, so $C^{*}\left(M_{z}\right) \cong C\left(\sigma\left(M_{z}\right)\right)$, and the linear functional $\eta$, given by

$$
\eta(f):=\left\langle f\left(M_{z}\right)(1+\mathcal{N}), 1+\mathcal{N}\right\rangle \quad\left(f \in C\left(\sigma\left(M_{z}\right)\right)\right),
$$

is positive. Thus, there exists a positive Borel measure $\mu$, with supp $\mu \subseteq \sigma\left(M_{z}\right)$, such that $\eta(f)=\int f d \mu$. Then

$$
\int \bar{z}^{i} z^{j} d \mu=\eta\left(\bar{z}^{i} z^{j}\right)=\left\langle M_{z}^{* i} M_{z}^{j}(1+\mathcal{N}), 1+\mathcal{N}\right\rangle=\left\langle z^{j}+\mathcal{N}, z^{i}+\mathcal{N}\right\rangle=\left\langle z^{j}, z^{i}\right\rangle_{M}=\gamma_{i j}
$$


By Proposition 5.8,

$$
\text { card supp } \mu=\operatorname{rank} M=r<\infty,
$$

so that $\left\{1, z, \ldots, z^{r-1}\right\}$ is a basis for $L^{2}(\mu)$, from which one derives that $\left\{1, Z, \ldots, Z^{r-1}\right\}$ is independent in the column space $\mathcal{C}_{M(r-1)}$, and a fortiori independent in the column space $\mathcal{C}_{M}$. Since $r=\operatorname{dim} \mathcal{C}_{M}$, we see that $\left\{1, Z, \ldots, Z^{r-1}\right\}$ is a basis for $\mathcal{C}_{M}$. Therefore, there exist unique scalars $\alpha_{0}, \ldots, \alpha_{r-1}$ such that $Z^{r}=\alpha_{0} 1+\ldots+\alpha_{r-1} Z^{r-1}$ in $\mathcal{C}_{M}$. This implies that $\operatorname{supp} \mu \subseteq \mathcal{Z}(p)$, where

$$
p(z):=z^{r}-\left(\alpha_{0}+\ldots+\alpha_{r-1} z^{r-1}\right) .
$$

Now $r=$ card supp $\mu \leq \operatorname{card} \mathcal{Z}(p) \leq r$, so $p$ has exactly $r$ distinct roots, say $z_{0}, \ldots, z_{r-1}$, and $\operatorname{supp} \mu=\mathcal{Z}(p)$. Thus $\mu=\sum_{i=0}^{r-1} \rho_{i} \delta_{z_{i}}$, where $\rho_{0}, \ldots, \rho_{r-1}$ are uniquely determined by the Vandermonde equation (5.1).

Remark 5.10. Observe that $\sigma\left(M_{z}\right)=\mathcal{Z}(p)$ and $r=\min \left\{j: Z^{j} \in\left\langle 1, \ldots, Z^{j-1}\right\rangle\right\}$.

\section{Flatness for moment matrices}

Definition 6.1. Given $\gamma: \gamma_{00}, \gamma_{01}, \gamma_{10}, \ldots, \gamma_{0,2 n}, \ldots, \gamma_{2 n, 0}$, with $\gamma_{00}>0$ and $\gamma_{j i}=$ $\bar{\gamma}_{i j}$, we say that $\gamma$ is flat if the columns corresponding to monomials of total degree $n$ are linear combinations of columns corresponding to monomials of total degree at most $n-1$.

Our first result establishes that flat data give rise to flat extensions of the associated moment matrix.

Theorem 6.2 ([CuF4, Theorem 5.4]). If $\gamma$ is flat and $M(n) \geq 0$, then $M(n)$ admits $a$ unique flat extension of the form $M(n+1)$.

The proof of Theorem 6.2 is lengthy, and it requires, among other things, extensive use of Theorem 5.2. As a corollary, we establish the existence of representing measures, and the equivalence of (v) and (vi) in Conjecture 4.1.

COROllary 6.3 ([CuF4, Corollary 5.12]). If $\gamma$ is flat and $M(n) \geq 0$, then $M(n)$ admits $a$ unique positive extension of the form $M(\infty)$, and this is a flat extension of $M(n)$.

Theorem 6.4 ([CuF4, Teorem 5.13]). The truncated moment sequence $\gamma$ has a rank $M(n)$-atomic representing measure if and only if $M(n) \geq 0$ and $M(n)$ admits a flat extension $M(n+1)$.

Corollary 6.5 ([CuF4, Corollary 5.14]). If $\gamma$ is flat and $M(n) \geq 0$, then there exists a positive Borel measure $\mu$ having moments of all orders, and $\mu$ is rank $M(n)$-atomic.

Corollary 6.6 ([CuF4, Corollary 5.15]). Assume $M(n) \geq 0$ and that the analytic columns of $M(n)$ are linearly dependent. Let $r:=\min \left\{k \geq 1: Z^{k} \in\left\langle 1, \ldots, Z^{k-1}\right\rangle\right\}$. Then there exists a positive Borel measure $\mu$ if and only if $\left\{1, \ldots, Z^{r-1}\right\}$ spans $\mathcal{C}_{M(n)}$. In this case, write $Z^{r}=a_{0} 1+\ldots+a_{r-1} Z^{r-1}$. Then $p(z):=z^{r}-\left(a_{0}+\ldots+a_{r-1} z^{r-1}\right)$ has $r$ distinct roots, $z_{0}, \ldots, z_{r-1}$, and $\mu$ is unique, given by $\mu=\sum_{i=0}^{r-1} \rho_{i} \delta_{z_{i}}$, where the densities are calculated using (5.1). 
Our approach leads to a concrete algorithm to compute explicitly the unique representing measure associated with a flat positive moment matrix, which we now present.

Algorithm 6.7. Given $M(n)$, let $r=\operatorname{rank} M(n)$.

STEP 1. If $r \leq n$ then $\left\{1, Z, \ldots, Z^{n}\right\}$ is linearly dependent, so there exists a unique positive Borel measure $\mu$, calculated as outlined before.

STEP 2. If $r>n$, use linear algebra to compute the polynomials $p_{i j} \in \mathcal{P}_{n-1}$ such that $\bar{Z}^{i} Z^{j}=p_{i j}(Z, \bar{Z})$ for $i+j=n$. Then use the $p_{i j}$ 's to compute the unique flat extension $M(n+1)$.

STEP 3. Compare $r$ and $n+1$. In case $r=n+1$, go to Step 1 . Else, $r>n+1$, and repeat Step 2.

6.1. Three applications of Theorem 6.4. We first consider the quadratic moment problem, corresponding to the case of initial data $\gamma: \gamma_{00}, \gamma_{01}, \gamma_{10}, \gamma_{02}, \gamma_{11}, \gamma_{20}, \gamma_{00}>0$, $\gamma_{i j}=\bar{\gamma}_{i j}(0 \leq i+j \leq 2)$. The proof of the following theorem, which we will omit, makes use of the equivalence of (v) and (vi) in Conjecture 4.1 (Theorem 6.4); that is, we set out to build a flat extension $M(2)$ based on the positivity of $M(1)$. For $n=3$, we shall see later (Section 8) that positivity alone does not suffice, for there exists $\gamma$ such that $M(3)(\gamma)$ is positive and invertible but admitting no representing measure. Whether such a phenomenon can arise when $n=2$ is a puzzling question.

Theorem 6.8 ([CuF4, Theorem 6.1]). Let $r:=\operatorname{rank} M(1)$. The following are equivalent.

(i) $\gamma$ has a representing measure;

(ii) $\gamma$ has an r-atomic representing measure;

(iii) $M(1) \geq 0$.

In this case,

(i) if $r=1$, there exists a unique representing measure;

(ii) if $r=2$, the 2-atomic representing measures are parameterized by a line;

(iii) if $r=3$, the 3 -atomic representing measures contain a sub-parameterization by a circle.

Rem ark 6.9. Observe that unlike the truncated Hamburger moment problem, it is quite possible to have infinitely many solutions when the associated moment matrix is singular.

Problem 6.10. Consider $\gamma: \gamma_{00}, \gamma_{01}, \gamma_{10}, \ldots, \gamma_{04}, \gamma_{13}, \gamma_{22}, \gamma_{31}, \gamma_{40}, \gamma_{00}>0, \gamma_{i j}=\bar{\gamma}_{i j}$ $(0 \leq i+j \leq 4)$ and assume that $M(2)(\gamma) \geq 0$. Does there exist a flat extension $M(3)(\gamma)$ ?

Next, we look at the case of $\bar{Z}=\alpha 1+\beta Z$; our aim is to show that $M(n)$ admits always a flat extension $M(n+1)$, and therefore there exists a finitely atomic representing measure $\mu$ for $\gamma$. Our ploy is to define a suitable block $B$ of the form $M(n) W$ so that

$$
M(n+1) \equiv\left(\begin{array}{cc}
M(n) & B \\
B^{*} & C
\end{array}\right)
$$

fulfills the conditions in Smul'jan's positivity criterion [CuF4, Proposition 2.2]. 
Theorem 6.11 ([CuF5, Theorem 2.1]). Assume that $M(n)$ has the property (RG) and that $\bar{Z}=\alpha 1+\beta Z$. Then $M(n)$ admits a flat extension $M(n+1)$.

Sketch of proof. Without loss of generality, we can assume that the columns 1 and $Z$ are linearly independent. Since $\bar{Z}=\alpha 1+\beta Z$, we must have

$$
Z=\bar{\alpha} 1+\bar{\beta} \bar{Z}=\bar{\alpha} 1+\bar{\beta}(\alpha 1+\beta Z)=(\bar{\alpha}+\bar{\beta} \alpha) 1+|\beta|^{2} Z .
$$

Then

$$
\bar{\alpha}+\bar{\beta} \alpha=0, \quad|\beta|=1 .
$$

Assume for a moment that $n=1$, and consider the column $Z^{2}$. We know that $\left\langle Z^{2}, 1\right\rangle$ must equal $\gamma_{02}$, and that

$$
\left\langle Z^{2}, Z\right\rangle=\langle Z, \bar{Z} Z\rangle=\langle Z,(\alpha 1+\beta Z) Z\rangle=\bar{\alpha}\langle Z, Z\rangle+\bar{\beta}\left\langle Z, Z^{2}\right\rangle=\bar{\alpha}\langle Z, Z\rangle+\bar{\beta} \overline{\left\langle Z^{2}, Z\right\rangle}
$$

In view of (6.1), (6.2) becomes

$$
\alpha\left\langle Z^{2}, Z\right\rangle=|\alpha|^{2}\langle Z, Z\rangle+\alpha \bar{\beta} \overline{\left\langle Z^{2}, Z\right\rangle}=|\alpha|^{2}\langle Z, Z\rangle-\bar{\alpha} \overline{\left\langle Z^{2}, Z\right\rangle},
$$

that is, $2 \operatorname{Re}\left(\alpha\left\langle Z^{2}, Z\right\rangle\right)=|\alpha|^{2}\langle Z, Z\rangle$. Therefore,

$$
\alpha\left\langle Z^{2}, Z\right\rangle=\frac{1}{2}|\alpha|^{2}\langle Z, Z\rangle+i t
$$

for some $t \in \mathbb{R}$. Observe also that

$$
\left\langle Z^{2}, \bar{Z}\right\rangle=\left\langle Z^{2}, \alpha 1+\beta Z\right\rangle=\bar{\alpha}\left\langle Z^{2}, 1\right\rangle+\bar{\beta}\left\langle Z^{2}, Z\right\rangle,
$$

and since we must necessarily have $\bar{Z} Z=\alpha Z+\beta Z^{2}$ and $\bar{Z}^{2}=\alpha \bar{Z}+\beta \bar{Z} Z$ (by property $(\mathrm{RG})$ ), we see that the choice of $\left\langle Z^{2}, Z\right\rangle$ fully determines the remaining entries of $M(2)$. Of course, one must still prove that the block $C$ is a Toeplitz matrix before concluding that this extension of $M(1)$ is a moment matrix. For $n>1$ the above idea still works, and leads to the definition of $Z^{n+1}$, which is the key to the construction of the block $M(n) W$. The techniques needed are much more sophisticated, and require several applications of Theorem 5.2.

To end this section we deal with the construction of flat extensions for $Z^{k} \in$ $\left\langle\bar{Z}^{i} Z^{j}\right\rangle_{0 \leq i+j \leq k-1}$.

\section{Case $1 \leq k \leq[n / 2]+1$}

Theorem 6.12 ([CuF5, Theorem 3.1]). Suppose $M(n)$ is positive and satisfies (RG). If $Z^{k}=p(Z, \bar{Z}), p \in \mathcal{P}_{k-1}$, then $M(n)$ admits a unique flat extension $M(n+1)$.

Remark 6.13. For $n$ odd, or $n$ even and $k<[n / 2]+1, M(n)$ is actually flat. However, this is not true for general $n$ and $k$.

ExAmple 6.14. We show that when $n$ is even and $k=[n / 2]+1$, it is possible to have a non-flat $M(n)$. Let $n=2$ and let

$$
M(2):=\left(\begin{array}{cccccc}
1 & 0 & 0 & 0 & 1 & 0 \\
0 & 1 & 0 & \alpha & \bar{\alpha} & \bar{\beta} \\
0 & 0 & 1 & \beta & \alpha & \bar{\alpha} \\
0 & \bar{\alpha} & \bar{\beta} & |\alpha|^{2}+|\beta|^{2} & \bar{\alpha}^{2}+\bar{\beta} \alpha & 2 \bar{\alpha} \bar{\beta} \\
1 & \alpha & \bar{\alpha} & \alpha^{2}+\beta \bar{\alpha} & |\alpha|^{2}+|\beta|^{2} & \bar{\alpha}^{2}+\bar{\beta} \alpha \\
0 & \beta & \alpha & 2 \alpha \beta & \alpha^{2}+\beta \bar{\alpha} & |\alpha|^{2}+|\beta|^{2}
\end{array}\right)
$$


Note that $Z^{2}=\alpha Z+\beta \bar{Z}$ and $\bar{Z}^{2}=\bar{\alpha} \bar{Z}+\bar{\beta} Z$. Since $\left\{1, Z, Z^{2}\right\}$ is linearly independent, $M(2)$ satisfies (RG) (vacuously!). Now,

$$
M(2) \geq 0 \Leftrightarrow|\beta|^{2} \geq 1+|\alpha|^{2}
$$

moreover, if $|\beta|^{2}>1+|\alpha|^{2}$ then $M(2)$ is not flat (since $\operatorname{rank} M(2)=4>\operatorname{rank} M(1)$ ). In this case, the unique 4 -atomic representing measure is of the form $\mu=\sum_{i=0}^{3} \rho_{i} \delta_{z_{i}}$, where the atoms $z_{0}, \ldots, z_{3}$ are the four distinct roots of

$$
z^{4}=2 \alpha z^{3}+\left(\beta \bar{\alpha}-\alpha^{2}\right) z^{2}+\beta\left(|\beta|^{2}-|\alpha|^{2}\right) z .
$$

Case $n \geq k>[n / 2]+1$. From $Z^{k}=p(Z, \bar{Z})$ we can derive a relation for $Z^{n+1}$, which a fortiori defines the corner $B$ of $M(n+1)$. If $\operatorname{Ran} B \nsubseteq \mathcal{C}_{M(n)}$, then there is no flat extension of $M(n)$; otherwise, let $W$ be such that $B=M(n) W$. Then $M(n)$ admits a flat extension $M(n+1)$ if and only if $W^{*} M(n) W$ is a Toeplitz matrix.

Re mark 6.15 . For $n=2$, we can now analyze the moment matrix $M(2)$ as follows: if the columns $1, Z, \bar{Z}$ are linearly dependent then Theorem 6.11 combined with basic facts from Section 3 will allow us to obtain a representing measure. If $\{1, Z, \bar{Z}\}$ is linearly independent and $\left\{1, Z, \bar{Z}, Z^{2}\right\}$ or $\left\{1, Z, \bar{Z}, \bar{Z}^{2}\right\}$ is linearly dependent, then Theorem 6.12 applies. Thus, the study of Problem 6.10 reduces to consideration of the cases when $\operatorname{rank} M(2) \geq 4$, with both $\left\{1, Z, \bar{Z}, Z^{2}\right\}$ and $\left\{1, Z, \bar{Z}, \bar{Z}^{2}\right\}$ linearly independent.

7. The case of independent analytic columns. When the analytic columns are linearly independent, the TCMP is unsolved; in particular, this occurs when $M(n)$ is positive and invertible, and $n>1$. The difficulty can best be explained by analogy with the real case. Since a positive singular Hankel matrix $H(k)$ always satisfies

$$
T^{r+s}=a_{0} T^{s}+\ldots+a_{r-1} T^{r+s-1} \quad(0 \leq s \leq k-r-1),
$$

the criterion for a positive Hankel extension $H(k+1)$ is simply

$$
T^{k}=a_{0} T^{k-r}+\ldots+a_{r-1} T^{k-1}
$$

in this case, there is a unique flat extension $H(k+1)$, determined by

$$
T^{k+1}:=a_{0} T^{k-r+1}+\ldots+a_{r-1} T^{k} .
$$

In the nonsingular case, we can choose $\gamma_{2 k+1}$ arbitrarily, and use the unique $\gamma_{2 k+2}$ determined from $\gamma$ and $\gamma_{2 k+1}$ by the flatness requirement.

For $M(n) \geq 0$ satisfying ( $\mathrm{RG})$, to produce a flat extension $M(n+1)$ we must choose the entire block $M[n, n+1]$ compatible with positivity, and in such a way that $M[n+$ $1, n+1]$ (which is then uniquely determined by the flatness requirement) is a Toeplitz matrix. Being able to achieve both conditions simultaneously is what makes the problem especially hard.

8. An invertible moment matrix admitting no representing measure. The aim of this section is to exhibit a positive invertible moment matrix $M(3)$ admitting no representing measure. Our construction is based on a 1979 result of K. Schmüdgen [Sch1] on the existence of a polynomial $q \in \mathbb{R}[x, y]$ of total degree 6 which is nonnegative on $\mathbb{R}^{2}$ but cannot be written as a sum of squares, a fact previously established abstractly by 
D. Hilbert [Hil] using tools from algebraic geometry. (The reader can find another such concrete polynomial in $[\mathrm{BCJ}]$.

Theorem 8.1 ([Sch1]). (1) The polynomial

$$
q(x, y):=200\left(x^{3}-4 x\right)^{2}+200\left(y^{3}-4 y\right)^{2}+\left(y^{2}-x^{2}\right) x(x+2)\left[x(x-2)+2\left(y^{2}-4\right)\right]
$$

is nonnegative on $\mathbb{R}^{2}$, but cannot be written as a sum of squares.

(2) There exists a positive linear functional $F$ on $\mathbb{C}[x, y]$ with $F(q)<0$. Thus, $F$ cannot be represented as integration with respect to a positive measure with support in $\mathbb{R}^{2}$.

$F$ is defined first on the space $\mathbb{C}_{6}[x, y]$ (the complex polynomials of total degree at most 6 ) as a linear combination of evaluation functionals, and then extended to all of $\mathbb{C}[x, y]$ :

$$
F(p):=32 \sum_{i=1}^{8} p\left(A_{i}\right)+p\left(B_{1}\right)+p\left(B_{2}\right)-p\left(A_{9}\right) \quad\left(p \in \mathbb{C}_{6}[x, y]\right)
$$

where

$$
\begin{array}{llll}
A_{1}:=(-2,-2), & A_{2}:=(0,-2), & A_{3}:=(2,-2), & A_{4}:=(-2,0), \\
A_{5}:=(0,0), & A_{6}:=(-2,2), & A_{7}:=(0,2), & A_{8}:=(2,2), \\
A_{9}:=(2,0), & B_{1}:=\left(\frac{1}{100}, 0\right), & B_{2}:=\left(0, \frac{1}{100}\right) . &
\end{array}
$$

We let

$$
\gamma_{k \ell}:=F\left((x-i y)^{k}(x+i y)^{\ell}\right) \quad(0 \leq k+\ell \leq 6)
$$

observe that

$$
\gamma_{k \ell}=\sum_{r=0}^{k} \sum_{s=0}^{\ell}(-1)^{k-r} i^{k+\ell-r-s}\left(\begin{array}{l}
k \\
i
\end{array}\right)\left(\begin{array}{l}
\ell \\
j
\end{array}\right) F\left(x^{r+s} y^{k+\ell-r-s}\right),
$$

and that

$$
F\left(x^{r} y^{s}\right)= \begin{cases}257, & r=0 \text { and } s=0 \\ 96\left[(-2)^{s}+2^{s}\right]+10^{-2 s}, & r=0 \text { and } s \geq 1 \\ 32\left[3(-2)^{r}+2^{r+1}\right]+10^{-2 r}-2^{r}, & r \geq 1 \text { and } s=0 \\ {\left[1+(-1)^{r}\right]\left[1+(-1)^{s}\right] 2^{r+s+5},} & r \geq 1 \text { and } s \geq 1\end{cases}
$$

The associated matrix $M(3)$ is built using the following values:

$$
\begin{gathered}
\gamma_{00}=257 ; \quad \gamma_{01}=10^{-2}(1-6599 i) ; \quad \gamma_{02}=132 ; \quad \gamma_{11}=\frac{7020001}{5000} ; \\
\gamma_{03}=10^{-6}(1+263999999 i) ; \quad \gamma_{12}=\bar{\gamma}_{03} ; \quad \gamma_{04}=\frac{333599999999}{50000000} ; \quad \gamma_{13}=528 ; \\
\gamma_{22}=\frac{485600000001}{50000000} ; \quad \gamma_{05}=10^{-10}(1-10559999999999 i) ; \quad \gamma_{14}=\bar{\gamma}_{05} ; \gamma_{23}=\gamma_{05} ; \\
\gamma_{06}=2112 ; \quad \gamma_{15}=-\frac{29727999999999999}{500000000000} ; \quad \gamma_{24}=\gamma_{06} ; \quad \gamma_{33}=\frac{35808000000000001}{500000000000} .
\end{gathered}
$$

Theorem $8.2([\mathrm{CuF} 5$, Section 4$])$. For $\gamma$ as above, $M(3)(\gamma)$ is positive and invertible. However, $\gamma$ admits no representing measure.

Proof. By the Nested Determinant Test, $M(3) \geq 0$ and $\operatorname{det} M(3)>0$. Since the presence of a representing measure for $\gamma$ would immediately give a corresponding measure for $F$, it follows that $M(3)(\gamma)$ cannot admit a representing measure. 
9. An application to numerical analysis. Consider the Lebesgue measure on $[0,1] \times[0,1]$, and the collection

$$
\widetilde{\gamma}_{i j}:=\int_{0}^{1} \int_{0}^{1} x^{i} y^{j} d x d y \quad(0 \leq i+j \leq 2) .
$$

Problem 9.1. Find a 3 -atomic measure $\mu$ supported in $[0,1] \times[0,1]$ such that

$$
\widetilde{\gamma}_{i j}:=\int_{[0,1] \times[0,1]} x^{i} y^{j} d \mu(x, y) \quad(0 \leq i+j \leq 2) .
$$

The existence of such a measure was established abstractly by V. Tchakaloff [Tch] in 1957 and concretely in A. H. Stroud's text [Str]. We shall see that our techniques will also allow us to exhibit one such measure. First, we must complexify the problem.

On the space $\mathbb{C}[x, y]_{2}$ of complex polynomials of total degree at most 2 , let $\widetilde{\varphi}$ be the complex linear functional induced by $\widetilde{\gamma}$, i.e., $\widetilde{\varphi}\left(t_{1}^{k_{1}} t_{2}^{k_{2}}\right):=\widetilde{\gamma}_{\left(k_{1}, k_{2}\right)}, 0 \leq k_{1}+k_{2} \leq 2$. For $0 \leq j+\ell \leq 2$ define $\gamma_{j \ell}:=\widetilde{\varphi}\left(\left(t_{1}-i t_{2}\right)^{j}\left(t_{1}+i t_{2}\right)^{\ell}\right)$. In our case, $\gamma_{00}=1, \gamma_{01}=\widetilde{\gamma}_{(1,0)}+$ $i \widetilde{\gamma}_{(0,1)}=(1+i) / 2, \gamma_{02}=\widetilde{\gamma}_{(2,0)}+2 i \widetilde{\gamma}_{(1,1)}-\widetilde{\gamma}_{(0,2)}=i / 2$, and $\gamma_{11}=\widetilde{\gamma}_{(2,0)}+\widetilde{\gamma}_{(0,2)}=2 / 3$. To build

$$
M(2)=\left(\begin{array}{cc}
M(1) & M(1) W \\
W^{*} M(1) & C
\end{array}\right)
$$

we set $\gamma_{12}:=a+b i$ and $\gamma_{03}:=c+d i$. The range condition, $\left(\gamma_{02} \gamma_{12} \gamma_{03}\right)^{T} \in \operatorname{Ran} M(1)$ leads at once to three coefficients $c_{0}, c_{1}, c_{2}$ such that $M(1)\left(\begin{array}{lll}c_{0} & c_{1} & c_{2}\end{array}\right)^{T}=\left(\begin{array}{lll}\gamma_{02} & \gamma_{12} & \gamma_{03}\end{array}\right)^{T}$. For $M(2)$ to be flat we must necessarily have $C=W^{*} M(1) W$, which implies that $Z^{2}=$ $c_{0} 1+c_{1} Z+c_{2} \bar{Z}$ and, by recursiveness, leads to the expression $Z^{3}=c_{0} Z+c_{1} Z^{2}+c_{2} \bar{Z} Z$, which gives $\gamma_{23}$. The concrete condition for the flatness of $M(2)$ is the following:

$$
-49 / 36+5 a-6 a^{2}+5 b-6 b^{2}+3 c+6 c^{2}-3 d+6 d^{2}=0 .
$$

To find the support of $\mu$, we compute $\mathbf{v}:=\left(M(2)_{\text {anal }}\right)^{-1}\left(\gamma_{03} \gamma_{13} \gamma_{23}\right)^{T}$, where

$$
M(2)_{\text {anal }}:=\left(\begin{array}{lll}
\gamma_{00} & \gamma_{01} & \gamma_{02} \\
\gamma_{10} & \gamma_{11} & \gamma_{12} \\
\gamma_{20} & \gamma_{21} & \gamma_{22}
\end{array}\right) .
$$

We then solve the equation

$$
z^{3}=v_{0}+v_{1} z+v_{2} z^{2}
$$

and select $a, b, c$ to guarantee that $z_{0}, z_{1}, z_{2} \in[0,1] \times[0,1]$. This can indeed be done, and we obtain a measure $\mu=\rho_{0} \delta_{z_{0}}+\rho_{1} \delta_{z_{1}}+\rho_{2} \delta_{z_{2}}$, where $\rho_{0}=\rho_{1}=\rho_{2}=1 / 3, z_{0} \cong$ $.853553+.295876 i, z_{1} \cong .5+.908248 i$ and $z_{2} \cong .146447+.295876 i$.

For degree 4 , our techniques predict a quadrature formula with a 6 -atomic measure, while Tchakaloff's work guarantees the existence (abstractly) of a 15-atomic measure. B. Reznick has recently obtained one such 6-atomic measure [Rez2], using his previous work on orthogonal polynomials [Rez1]. We are currently attempting to use our methods to obtain another such measure.

Some of the calculations in this section were obtained with the help of the software tool Mathematica [Wol]. 
10. Conclusion. We have seen how positivity and extension properties of finite matrices can lead to existence and uniqueness results for truncated moment problems, and to adequate (although not completely satisfactory) descriptions of notions such as quadratic hyponormality. Our methods can be extended to the multidimensional moment problem and to truncated moment problems in one or several real or complex variables ([CuF4, Chapter 7]), and they allow us to deal with the Subnormal Completion Problem for commuting multivariable weighted shifts (for a definition and basic of this class we refer the reader to $[\mathrm{JeL}])$; an example of such an application can be found in $[\mathrm{CuF} 4$, Section 6.2]. Needless to say, there are limitations to the scope of our techniques, and they only represent a small sample of the wide range of investigations that have been carried out for moment problems (truncated and full) in the last several years, whether they involve methods from operator theory or not. The reader will find vast information on other approaches to the topics discussed in this article in $[\mathrm{AK}],[\mathrm{Akh}],[\mathrm{And}],[\mathrm{Atz}]$, [Ber], [BCJ], [BeM], [Cas], [Fia], [Fug], [Hau], [Hav1], [Hav2], [KrN], [Lan], [Li], [McCY], [McG], [Nar], [Pu1], [Pu2], [Pu3], [Pu4], [Pu5], [Rez1], [Sar], [Sch2], [ShT], [StSz1], [StSz2], [StSz3], [StSz4], [Sza1] and [Sza2].

Note. In [Pru], B. Prunaru has announced a positive solution to Problem 2.6.

\section{References}

[Agl] J. Agler, Hypercontractions and subnormality, J. Operator Theory 13 (1985), 203-217.

[AK] N. I. Ahiezer and M. G. Krein, Some Questions in the Theory of Moments, Transl. Math. Monographs 2, Amer. Math. Soc., Providence, 1962.

[Akh] N. I. Akhiezer, The Classical Moment Problem, Hafner, New York, 1965.

[And] T. Ando, Truncated moment problems for operators, Acta Sci. Math. (Szeged) 31 (1970), 319-333.

[Atz] A. Atzmon, A moment problem for positive measures on the unit disc, Pacific J. Math. 59 (1975), 317-325.

[Ber] C. Berg, The multidimensional moment problem and semigroups, in: Moments in Mathematics, Proc. Sympos. Appl. Math. 37, Amer. Math. Soc., 1987, 110-124.

[BCJ] C. Berg, J. P. R. Christensen and C. U. Jensen, A remark on the multidimensional moment problem, Math. Ann. 223 (1979), 163-169.

[BeM] C. Berg and P. H. Maserick, Polynomially positive definite sequences, ibid. 259 (1982), 487-495

[Bra] J. Bram, Subnormal operators, Duke Math. J. 22 (1955), 75-94.

[Cas] G. Cassier, Problème des moments sur un compact de $\mathbf{R}^{n}$ et décomposition des polynômes à plusieurs variables, J. Funct. Anal. 58 (1984), 254-266.

[Con] J. B. Conway, Subnormal Operators, Pitman, London, $1981\left(^{1}\right)$.

[CoC] M. Cotlar and R. Cignoli, An Introduction to Functional Analysis, North-Holland, Amsterdam, 1974.

[Cu1] R. Curto, Quadratically hyponormal weighted shifts, Integral Equations Operator Theory 13 (1990), 49-66.

$\left({ }^{1}\right)$ Editorial note: See also J. B. Conway, The Theory of Subnormal Operators, Math. Surveys Monographs 36, Amer. Math. Soc., Providence, R.I., 1991. 
[Cu2] R. Curto, Joint hyponormality: A bridge between hyponormality and subnormality, in: Proc. Sympos. Pure Math. 51, Part 2, Amer. Math. Soc., 1990, 69-91.

[Cu3] - Polynomially hyponormal operators on Hilbert space, Rev. Un. Mat. Argentina 37 (1991), 29-56.

[CuF1] R. Curto and L. Fialkow, Recursively generated weighted shifts and the subnormal completion problem, Integral Equations Operator Theory 17 (1993), 202-246.

[CuF2] - - , Recursively generated weighted shifts and the subnormal completion problem, II, ibid. 18 (1994), 369-426.

[CuF3] -, 一, Recursiveness, positivity, and truncated moment problems, Houston J. Math. 17 (1991), 603-635.

[CuF4] - - , Solution of the truncated complex moment problem for flat data, Mem. Amer. Math. Soc. 119 (1996).

[CuF5] -, - Flat extensions of positive moment matrices: Relations in analytic or conjugate terms, in: Oper. Theory Adv. Appl., to appear.

[CuF6] - - - Flat extensions of positive moment matrices: Recursively generated relations, Mem. Amer. Math. Soc., to appear.

[CMX] R. Curto, P. Muhly and J. Xia, Hyponormal pairs of commuting operators, in: Oper. Theory Adv. Appl. 35, Birkhäuser, 1988, 1-22.

[CuP1] R. Curto and M. Putinar, Existence of non-subnormal polynomially hyponormal operators, Bull. Amer. Math. Soc. 25 (1991), 373-378.

[CuP2] -, 一, Nearly subnormal operators and moment problems, J. Funct. Anal. 115 (1993), 480-497.

[Fan] P. Fan, A note on hyponormal weighted shifts, Proc. Amer. Math. Soc. 92 (1984), $271-272$.

[Fia] L. Fialkow, Positivity, extensions and the truncated complex moment problem, in: Contemp. Math. 185, Amer. Math. Soc., 1995, 133-150.

[Fug] B. Fuglede, The multidimensional moment problem, Exposition. Math. 1 (1983), 47-65.

[Hal] P. R. Halmos, Normal dilations and extensions of operators, Summa Brasil. Math. 2 (1950), 124-134.

[Hau] F. Hausdorff, Momentprobleme für ein endliches Intervall, Math. Z. 16 (1923), $220-248$.

[Hav1] E. K. Haviland, On the momentum problem for distributions in more than one dimension, Amer. J. Math. 57 (1935), 562-568.

[Hav2] -, On the momentum problem for distributions in more than one dimension, Part II, ibid. 58 (1936), 164-168.

[Hil] D. Hilbert, Über die Darstellung definiter Formen als Summen von Formenquadraten, Math. Ann. 32 (1888), 342-350.

[JeL] N. Jewell and A. Lubin, Commuting weighted shifts and analytic function theory in several variables, J. Operator Theory 1 (1979), 207-223.

[Jos1] A. Joshi, Hyponormal polynomials of monotone shifts, Ph.D. dissertation, Purdue University, 1971.

[Jos2] - Hyponormal polynomials of monotone shifts, Indian J. Pure Appl. Math. 6 (1975), 681-686.

[KrN] M. G. Krein and A. Nudel'man, The Markov Moment Problem and Extremal Problems, Transl. Math. Monographs 50, Amer. Math. Soc., Providence, 1977. 
[Lan] H. Landau, Classical background of the moment problem, in: Moments in Mathematics, Proc. Sympos. Appl. Math. 37, Amer. Math. Soc., 1987, 1-15.

[Li] X. Li, On positive moment sequences, Ph.D. dissertation, Virginia Tech. Univ., 1993.

[McC] J. McCarthy, private communication.

[McCY] J. McCarthy and L. Yang, Subnormal operators and quadrature domains, preprint, 1995.

[McCP] S. McCullough and V. Paulsen, A note on joint hyponormality, Proc. Amer. Math. Soc. 107 (1989), 187-195.

[McG] J. L. McGregor, Solvability criteria for certain $N$-dimensional moment problems, J. Approx. Theory 30 (1980), 315-333.

[Mys] I. P. Mysovskikh, On Chakalov's Theorem, USSR Comp. Math. 15 (1975), 221-227.

[Nar] F. J. Narcowich, R-operators II. On the approximation of certain operator-valued analytic functions and the Hermitian moment problem, Indiana Univ. Math. J. 26 (1977), 483-513.

[Pru] B. Prunaru, Invariant subspaces for polynomially hyponormal operators, preprint, 1995.

[Pu1] M. Putinar, A two-dimensional moment problem, J. Funct. Anal. 80 (1988), 1-8.

[Pu2] - The L problem of moments in two dimensions, ibid. 94 (1990), 288-307.

[Pu3] - Positive polynomials on compact semi-algebraic sets, Indiana Univ. Math. J. 42 (1993), 969-984.

[Pu4] - Extremal solutions of the two-dimensional L-problem of moments, J. Funct. Anal. 136 (1996), 331-364.

[Pu5] -, Quadrature domains and hyponormal operators, lecture at SEAM XI, Georgia Tech. Univ., Atlanta, 1995.

[Pu6] -, On Tchakaloff's Theorem, preprint, 1995.

[Rez1] B. Reznick, Sums of even powers of real linear forms, Mem. Amer. Math. Soc. 463 (1992).

[Rez2] -, e-mail communication.

[Sar] D. Sarason, Moment problems and operators on Hilbert space, in: Moments in Mathematics, Proc. Sympos. Appl. Math. 37, Amer. Math. Soc., 1987, 54-70.

[Sch1] K. Schmüdgen, An example of a positive polynomial which is not a sum of squares of polynomials. A positive, but not strongly positive functional, Math. Nachr. 88 (1979), 385-390.

[Sch2] - The K-moment problem for semi-algebraic sets, Math. Ann. 289 (1991), 203-206.

[SeS] Z. Sebestyén and J. Stochel, Restrictions of positive self-adjoint operators, Acta Sci. Math. (Szeged) 55 (1991), 149-154.

[ShT] J. Shohat and J. Tamarkin, The Problem of Moments, Math. Surveys 1, Amer. Math. Soc., Providence, 1943.

[Smu] J. L. Smul'jan, An operator Hellinger integral, Mat. Sb. 91 (1959), 381-430 (in Russian).

[Sta] J. Stampfli, Which weighted shifts are subnormal, Pacific J. Math. 17 (1966), 367379 .

[StSz1] J. Stochel and F. H. Szafraniec, A characterization of subnormal operators, in: Spectral Theory of Linear Operators and Related Topics, Birkhäuser, 1984, 261-263.

[StSz2] - - - Unbounded weighted shifts and subnormality, Integral Equations Operator Theory 12 (1989), 146-153. 
[StSz3] J. Stochel and F. H. Szafraniec, On normal extensions of unbounded operators, III. Spectral properties, Publ. RIMS 25 (1989), 105-139.

[StSz4] -, 一, Algebraic operators and moments on algebraic sets, Portugal. Math. 51 (1994), $1-21$.

[Str] A. H. Stroud, Approximate Calculation of Multiple Integrals, Prentice-Hall, 1971.

[Sza1] F. H. Szafraniec, Boundedness of the shift operator related to positive definite forms: An application to moment problems, Ark. Mat. 19 (1981), 251-259.

[Sza2] -, Moments on compact sets, in: Prediction Theory and Harmonic Analysis, V. Mandrekar and H. Salehi (eds.), North-Holland, Amsterdam, 1983, 379-385.

[Tch] V. Tchakaloff, Formules de cubatures mécaniques à coefficients non négatifs, Bull. Sci. Math. 81 (1957), 123-134.

[Wol] Wolfram Research, Inc., Mathematica, Version 2.1, Champaign, Illinois, 1992. 